TRANSACTIONS OF THE

AMERICAN MATHEMATICAL SOCIETY

Volume 354, Number 3, Pages 1079-1116

S 0002-9947(01)02865-3

Article electronically published on November 2, 2001

\title{
TRACE THEOREMS FOR THREE-DIMENSIONAL, TIME-DEPENDENT SOLENOIDAL VECTOR FIELDS AND THEIR APPLICATIONS
}

\author{
A. FURSIKOV, M. GUNZBURGER, AND L. HOU
}

\begin{abstract}
We study trace theorems for three-dimensional, time-dependent solenoidal vector fields. The interior function spaces we consider are natural for solving unsteady boundary value problems for the Navier-Stokes system and other systems of partial differential equations. We describe the space of restrictions of such vector fields to the boundary of the space-time cylinder and construct extension operators from this space of restrictions defined on the boundary into the interior. Only for two exceptional, but useful, values of the spatial smoothness index, the spaces for which we construct extension operators is narrower than the spaces in which we seek restrictions. The trace spaces are characterized by vector fields having different smoothnesses in directions tangential and normal to the boundary; this is a consequence of the solenoidal nature of the fields. These results are fundamental in the study of inhomogeneous boundary value problems for systems involving solenoidal vector fields. In particular, we use the trace theorems in a study of inhomogeneous boundary value problems for the Navier-Stokes system of viscous incompressible flows.
\end{abstract}

\section{INTRODUCTION}

Solenoidal vector fields appear in numerous applications, including fluid mechanics, electromagnetics, superconductivity, etc. Solenoidal fields usually result from a conservation law (e.g., conservation of mass for incompressible flows), or gauge choices (e.g., for the vector magnetic potential in electromagnetics and superconductivity), or compatibility conditions (e.g., the vorticity field is, by its definition as the curl of the velocity, solenoidal). In this paper, we study the properties of traces of three-dimensional, time-dependent solenoidal vector fields. The results we derive are of use in the study of inhomogeneous boundary value problems for systems of partial differential equations which involve solenoidal vector fields. In fact, we apply them to inhomogeneous boundary value problems for the Navier-Stokes system for unsteady, viscous, incompressible flows.

Our own motivation arises from our investigations of drag reduction for a body immersed in a viscous, incompressible flow by controlling the velocity of the fluid on the boundary of the body; see [5], where the two-dimensional analog of this problem was studied. The derivation of an optimality system for this optimal control problem was reduced in [5] to proving the existence of a solution $\mathbf{w} \in \mathbf{W}$

Received by the editors September 29, 1999 and, in revised form, May 23, 2000 and March 19, 2001.

2000 Mathematics Subject Classification. Primary 46E35, 35K50, 76D05, 76D07.

(C)2001 American Mathematical Society 
for the following boundary value problem:

$$
\begin{aligned}
& \mathbf{N S}^{\prime}(\widehat{\mathbf{v}})(t, \mathbf{x}) \mathbf{w}(t, \mathbf{x})=\mathbf{f}(t, \mathbf{x}), \quad(t, \mathbf{x}) \in Q_{T}, \\
& \left.\mathbf{w}\right|_{\Sigma_{T}}=\mathbf{g},\left.\quad \mathbf{w}\right|_{t=0}=\mathbf{v}_{0} .
\end{aligned}
$$

Here, $\mathbf{N S}^{\prime}(\widehat{\mathbf{v}})$ is the derivative of the Navier-Stokes operator evaluated at an optimizer $\widehat{\mathbf{v}}, Q_{T}=(0, T) \times \Omega$ is a time-space cylinder on which (1.1) is posed, where $\Omega \subset \mathbb{R}^{2}$ is the spatial domain with boundary $\partial \Omega$, and $\Sigma_{T}=(0, T) \times \partial \Omega$ is the lateral surface of the cylinder $Q_{T}$. The solvability of the problem (1.1) should be proved for each $\mathbf{f} \in \mathbf{F}, \mathbf{g} \in \mathbf{G}$, and $\mathbf{v}_{0} \in \mathbf{W}_{0}$, where $\mathbf{F}, \mathbf{G}$, and $\mathbf{W}_{0}$ are appropriate function spaces. The correct choice of $\mathbf{W}, \mathbf{F}$, and $\mathbf{G}$ is very important because it is closely connected to the correct mathematical formulation of the original drag reduction problem.

In the two-dimensional case, the space $\mathbf{W}$ is generated naturally (see 5]) by the drag functional and is the "energy space"

$$
\mathbf{W}=\mathcal{V}^{(1)}\left(Q_{T}\right) \equiv\left\{\mathbf{v} \in L^{2}\left(0, T ; \mathbf{H}^{1}(\Omega): \partial_{t} \mathbf{v} \in L^{2}\left(0, T ; \mathbf{H}^{-1}(\Omega)\right), \operatorname{div} \mathbf{v}=0\right\},\right.
$$

where $H^{k}(\Omega), k=1,-1$, are the usual Sobolev spaces with smoothness index $k$ and $\mathbf{H}^{k}(\Omega)=\left[H^{k}(\Omega)\right]^{d}$, with $d$ denoting the space dimension. Evidently, $\mathbf{F}=\{\mathbf{f} \in$ $\left.L^{2}\left(0, T ; \mathbf{H}^{-1}(\Omega)\right): \operatorname{div} \mathbf{f}=0\right\}$ and

$$
\mathbf{G}=\text { the trace space on } \Sigma_{T} \text { of } \mathcal{V}^{(1)}\left(Q_{T}\right) \text {. }
$$

The trace space (1.2) was characterized in [5].

To formulate the optimal control problem correctly in three dimensions, we use an approach parallel to that used in two dimensions: we consider (1.1) defined for $\mathbf{x} \in \Omega \subset \mathbb{R}^{3}$. Since it is mathematically inappropriate to use $\mathcal{V}^{(1)}\left(Q_{T}\right)$ in the three-dimensional case, we look for the desired space $\mathbf{W}$ in the class of "energy-type spaces"

$$
\mathcal{V}^{(s)}\left(Q_{T}\right) \equiv\left\{\mathbf{v} \in L^{2}\left(0, T ; \mathbf{H}^{s}(\Omega)\right): \partial_{t} \mathbf{v} \in L^{2}\left(0, T ; \mathbf{H}^{s-2}(\Omega)\right), \operatorname{div} \mathbf{v}=0\right\} .
$$

It is well known (see [4) that the boundary value problem (1.1) with the homogeneous boundary condition $\mathbf{g}=\mathbf{0}$ is well posed with $\mathbf{w} \in \mathcal{V}^{(s)}\left(Q_{T}\right)$ and $\widehat{\mathbf{v}} \in \mathcal{V}^{(s)}\left(Q_{T}\right)$ for $s \geq 3 / 2$. In the inhomogeneous case, i.e., $\mathbf{g} \neq \mathbf{0}$, the well-posedness of (1.1) is still desired. Therefore, to set up a well-posed formulation of the optimal drag reduction problem in the three-dimensional case, we have to solve the following problem:

$$
\begin{aligned}
& \text { characterize the space of Dirichlet traces } \\
& \text { onto } \Sigma_{T} \text { for the } \text { space }^{(s)}\left(Q_{T}\right) \text {. }
\end{aligned}
$$

This paper is devoted to solving problem (1.3) for $s>1 / 2$ in three dimensions $(d=3)$. We point out that the case $\mathcal{V}^{(s)}\left(Q_{T}\right)$ with $s=3 / 2$ is the most appropriate for the drag reduction problem in the three-dimensional case. Nevertheless, we are forced to study problem (1.3) for all $s>1 / 2$. The main reason for this is that the cases $s=3 / 2$ and $s=5 / 2$ are singular. Below, we prove that when $s>1 / 2, s \neq 3 / 2$, and $s \neq 5 / 2$, the trace space $G^{s}\left(\Sigma_{T}\right)$ exists for the space $\mathcal{V}^{(s)}\left(Q_{T}\right)$, and we give the precise description of $G^{s}\left(\Sigma_{T}\right)$. In particular, we have the exact formula for $\|\cdot\|_{G^{s}\left(\Sigma_{T}\right)}$; see (2.10)-(2.12) below. In the cases $s=3 / 2$ and $s=5 / 2$, the spaces $G_{\ln }^{3 / 2}\left(\Sigma_{T}\right)$ and $G_{\ln }^{5 / 2}\left(\Sigma_{T}\right)$ where we seek restrictions to $\Sigma_{T}$ of functions belonging to $\mathcal{V}^{(3 / 2)}\left(Q_{T}\right)$ and $\mathcal{V}^{(5 / 2)}\left(Q_{T}\right)$, respectively, are wider than the spaces $G^{3 / 2}\left(\Sigma_{T}\right)$ and $G^{5 / 2}\left(\Sigma_{T}\right)$ for which we can construct continuous extension 
operators $R: G^{3 / 2}\left(\Sigma_{T}\right) \rightarrow \mathcal{V}^{(3 / 2)}\left(Q_{T}\right)$ and $R: G^{5 / 2}\left(\Sigma_{T}\right) \rightarrow \mathcal{V}^{(5 / 2)}\left(Q_{T}\right)$. Note that the norms of the spaces $G^{s}\left(\Sigma_{T}\right)$ and $G_{\ln }^{s}\left(\Sigma_{T}\right)$ for $s=3 / 2$ and $s=5 / 2$ differ in their Fourier representations by a certain logarithmic multiplier; compare (2.4) and (2.7) below.

The study of the optimal control problem mentioned earlier is a motivation as well as an application of the trace theorems obtained in this paper (however, optimal control problems will not be addressed in this paper). Obviously, there are many other applications of these trace results simply because, as was already mentioned, many physical problems involve divergence-free vector fields on domains with a boundary. As an illustration of an application of the trace theorems to be obtained in this paper, boundary value problems for the Stokes and Navier-Stokes equations will be considered in the last section.

The methods which were used in 5 to solve the problem (1.3) for $s=1$ in the two-dimensional case applied the theory of interpolation (see 9]). However, this approach is possible only for $s=1$; it cannot be generalized to the threedimensional case for $s>1 / 2$. Roughly speaking, the method worked out below consists of two parts. The first part is the proof of the trace theorem for scalar functions belonging to the space

$$
\mathcal{H}^{(s)}\left(Q_{T}\right)=\left\{\phi \in L^{2}\left(0, T ; H^{s}(\Omega)\right): \partial_{t} \phi \in L^{2}\left(0, T ; H^{s-2}(\Omega)\right)\right\} .
$$

With the help of the method of localization, this task is reduced to the derivation of some estimates for Fourier representations. Of course, this approach is well known; see, e.g., 9]. Moreover, the proof of these estimates is also well known for $s>5 / 2$. However, the cases of most interest in applications are $1 / 2<s \leq 5 / 2$.

The second part is to work out the localization and rectification method for solenoidal vector fields. To establish the restriction theorem when the smoothness index $s$ of $\mathcal{V}^{(s)}\left(Q_{T}\right)$ is small, we increase the smoothness by transition from a solenoidal vector field $\mathbf{u}$ to a field $\mathbf{v}$ such that $\operatorname{curl} \mathbf{v}=\mathbf{u}$, and then we express the traces of $\mathbf{u}$ in terms of of the traces of $\mathbf{v}$. To realize this approach, we develop further some results of 3 on the solvability of the system

$$
\operatorname{curl} \mathbf{v}=\mathbf{u}, \quad \operatorname{div} \mathbf{v}=0 \quad \text { for } \mathbf{x} \in \Omega,\left.\quad v_{n}\right|_{\partial \Omega}=0,
$$

where $v_{n}$ is the projection of the vector field $\mathbf{v}$ onto the unit outward-pointing normal $\mathbf{n}$.

To obtain the extension result we need to solve the following problem posed on the manifold $\partial \Omega$ :

$$
\left.(\operatorname{curl} \mathbf{v})\right|_{\partial \Omega}=\left.\mathbf{u}\right|_{\partial \Omega},
$$

where $\left.\mathbf{u}\right|_{\partial \Omega}$ is a given vector field defined on $\partial \Omega$. Since $\left.(\mathbf{c u r l} \mathbf{v})\right|_{\partial \Omega}$ can be easily expressed in terms of $\left.\mathbf{v}\right|_{\partial \Omega}$ and $\left.(\partial \mathbf{v} / \partial n)\right|_{\partial \Omega}$, we can understand (1.4) as an equation for the unknowns $\left.\mathbf{v}\right|_{\partial \Omega}$ and $\left.(\partial \mathbf{v} / \partial n)\right|_{\partial \Omega}$. We can rewrite (1.4) as an equation on the manifold $\partial \Omega$ in an invariant form using exterior differential forms. With the help of this curl problem, it is very convenient for us to transform the trace problem for solenoidal vector fields to the analogous problem for exterior differential forms. To make this transformation in a simple way, we are compelled to use only a special kind of local coordinates, the "orthogonal local coordinates." To solve (1.4), we may use well-known results on the solvability of the Laplace operator in the classes of differential forms defined on the manifold $\partial \Omega$; see [1] and [10]. 
Some notations are in order. Throughout, $\Omega$ is a bounded domain in $\mathbb{R}^{d}, \partial \Omega$ is the boundary of $\Omega$, and $\partial \Omega$ is a compact and closed $C^{\infty}$ manifold consisting of $J$ connected components $\left\{\Gamma_{j}\right\}$,

$$
\partial \Omega=\bigcup_{j=1}^{J} \Gamma_{j} \quad \text { and } \quad \Gamma_{i} \cap \Gamma_{j}=\phi \quad \text { whenever } i \neq j .
$$

$Q_{T}=(0, T) \times \Omega$ denotes a space-time cylinder and $\Sigma_{T}=(0, T) \times \partial \Omega$ is its lateral boundary. $\mathbf{n}$ denotes the unit outward-pointing normal vector on $\partial \Omega$ or on $\Sigma_{T}$.

\section{Formulation of the trace theorem}

In this section, we first recall some definitions of the function spaces which will be used in the sequel; then, we formulate the trace theorem.

2.1. Function spaces. We introduce the space

$$
S=S\left(\mathbb{R}^{d}\right)=\left\{u: \mathbf{x}^{\alpha} D^{\beta} u \in L^{2}\left(\mathbb{R}^{d}\right), \forall \alpha, \forall \beta\right\},
$$

where $\mathbf{x}=\left(x_{1}, \cdots, x_{d}\right) \in \mathbb{R}^{d}, \alpha=\left(\alpha_{1}, \cdots, \alpha_{d}\right)$ and $\beta=\left(\beta_{1}, \cdots, \beta_{d}\right)$ are multiindices with nonnegative components, $\mathbf{x}^{\alpha}=x_{1}^{\alpha_{1}} \cdots x_{d}^{\alpha_{d}}$, and

$$
D^{\beta}=\partial^{|\beta|} /\left(\partial x_{1}^{\beta_{1}} \cdots \partial x_{d}^{\beta_{d}}\right) .
$$

For $u \in S$, we define the Fourier transform

$$
\widehat{u}(\boldsymbol{\xi})=\mathcal{F} u(\boldsymbol{\xi})=(2 \pi)^{-d / 2} \int_{\mathbb{R}^{d}} e^{-i \mathbf{x} \cdot \boldsymbol{\xi}} u(\mathbf{x}) d \mathbf{x},
$$

where $\mathbf{x} \cdot \boldsymbol{\xi}=x_{1} \xi_{1}+\cdots+x_{d} \xi_{d}$. Then, the inverse Fourier transform gives

$$
u(\mathbf{x})=\overline{\mathcal{F}} \widehat{u}(\mathbf{x})=(2 \pi)^{-d / 2} \int_{\mathbb{R}^{d}} e^{i \mathbf{x} \cdot \boldsymbol{\xi}} \widehat{u}(\boldsymbol{\xi}) d \boldsymbol{\xi} .
$$

Recall that the Schwartz space $S^{\prime}\left(\mathbb{R}^{d}\right)$ of slowly growing distributions is defined as the dual space of $S\left(\mathbb{R}^{d}\right)$; for details see [6] and [9, Chap. I, 1.2]. The Fourier transform of $u \in S^{\prime}$ is defined by the formula

$$
\langle\mathcal{F} u, \phi\rangle=\langle u, \overline{\mathcal{F}} \phi\rangle \quad \forall \phi \in S\left(\mathbb{R}^{d}\right),
$$

where $\langle\cdot, \cdot\rangle$ is the duality between $S^{\prime}$ and $S$ which is generated by the scalar product in the space $L^{2}\left(\mathbb{R}^{d}\right)$ of complex-valued functions.

For $s \in \mathbb{R}$, the Sobolev space $H^{s}\left(\mathbb{R}^{d}\right)$ is defined as follows:

$$
H^{s}\left(\mathbb{R}^{d}\right)=\left\{u \in S^{\prime}\left(\mathbb{R}^{d}\right):\left(1+|\boldsymbol{\xi}|^{2}\right)^{s / 2} \widehat{u} \in L^{2}\left(\mathbb{R}^{d}\right)\right\}
$$

with the norm defined by

$$
\|u\|_{H^{s}\left(\mathbb{R}^{d}\right)}^{2}=\int_{\mathbb{R}^{d}}\left(1+|\boldsymbol{\xi}|^{2}\right)^{s}|\widehat{u}(\boldsymbol{\xi})|^{2} d \boldsymbol{\xi} .
$$

On the closure $\bar{G}$ of a domain $G$, we introduce the following subspace of $H^{s}\left(\mathbb{R}^{d}\right)$ :

$$
H \frac{s}{G}\left(\mathbb{R}^{d}\right)=\left\{u \in H^{s}\left(\mathbb{R}^{d}\right): \operatorname{supp} u \subset \bar{G}\right\},
$$

where $\operatorname{supp} u$ is the support of the function $u$. For a domain $\Omega$, we denote $\Omega^{\prime}=$ $\mathbb{R}^{d} \backslash \Omega$. For $s \in \mathbb{R}$, the Sobolev space $H^{s}(\Omega)$ of functions defined on $\Omega$ is determined as follows (see [7] and [13]):

$$
H^{s}(\Omega)=H^{s}\left(\mathbb{R}^{d}\right) / H_{\Omega^{\prime}}^{s}\left(\mathbb{R}^{d}\right) .
$$


General definitions of quotient spaces of Banach spaces and (2.1) imply that there exists an extension operator $E: H^{s}(\Omega) \rightarrow H^{s}\left(\mathbb{R}^{d}\right)$ and

$$
\|u\|_{H^{s}(\Omega)}=\inf _{E}\|E u\|_{H^{s}\left(\mathbb{R}^{d}\right)}
$$

where the infimum is taken over all extension operators $E$.

We suppose that the boundary $\partial \Omega$ of a domain $\Omega \subset \mathbb{R}^{d}$ is a closed manifold of dimension $d-1$. Then, by the definition of a manifold, there exist a finite covering $\left\{U_{j}\right\}$ of $\partial \Omega$ and diffeomorphisms $\delta_{j}: U_{j} \rightarrow B=\left\{\mathbf{x} \in \mathbb{R}^{d}:|\mathbf{x}|<1\right\}$ such that, on $U_{i} \cap U_{j}(\neq \emptyset), \delta_{i} \circ \delta_{j}^{-1}$ is a $C^{\infty}$ map. Let $\left\{\phi_{j}\right\}$ be a partition of unity subordinate to $\left\{U_{j}\right\}$. Then, the norm $\|\cdot\|_{H^{s}(\partial \Omega)}$ is defined by the formula

$$
\|u\|_{H^{s}(\partial \Omega)}^{2}=\sum_{j}\left\|\phi_{j} u \circ \delta_{j}^{-1}\right\|_{H^{s}\left(\mathbb{R}^{d-1}\right)}^{2}
$$

and the Sobolev space $H^{s}(\partial \Omega)$ is determined as the space of distributions defined on $\partial \Omega$ which possess the finite norm (2.3); for details, see [9].

We will use the space $\mathbf{V}^{s}(\Omega)$ of solenoidal vector fields which is defined as follows:

$$
\mathbf{V}^{s}(\Omega)=\left\{\mathbf{v}=\left(v_{1}, \cdots, v_{d}\right) \in\left[H^{s}(\Omega)\right]^{d}: \operatorname{div} \mathbf{v}=0\right\} .
$$

The main object of our investigation will be spaces of functions that depend on both $\mathbf{x}$ and the time $t$. If $(t, \mathbf{x}) \in \mathbb{R}^{d+1}$, then the Fourier transform $\widehat{u}(\tau, \boldsymbol{\xi})$ is defined as follows:

$$
\widehat{u}(\tau, \boldsymbol{\xi})=\mathcal{F} u(\tau, \boldsymbol{\xi})=(2 \pi)^{-(d+1) / 2} \int_{\mathbb{R}^{d+1}} e^{-i(t \tau+\mathbf{x} \cdot \boldsymbol{\xi})} u(t, \mathbf{x}) d \mathbf{x} d t .
$$

We introduce the Schwartz space $S^{\prime}\left(\mathbb{R}^{d+1}\right)$ and define

$$
\mathcal{H}^{(s)}\left(\mathbb{R}^{d+1}\right)=\left\{u(t, \mathbf{x}) \in S^{\prime}\left(\mathbb{R}^{d+1}\right):\|u\|_{\mathcal{H}^{(s)}\left(\mathbb{R}^{d+1}\right)}<\infty\right\},
$$

where

$$
\begin{aligned}
& \|u\|_{\mathcal{H}^{(s)}\left(\mathbb{R}^{d+1}\right)}^{2} \\
& \quad \equiv \int_{\mathbb{R}^{d+1}}\left[\left(1+|\boldsymbol{\xi}|^{2}\right)^{s}+\left(1+|\tau|^{2}\right)\left(1+|\boldsymbol{\xi}|^{2}\right)^{s-2}\right]|\widehat{u}(\tau, \boldsymbol{\xi})|^{2} d \boldsymbol{\xi} d \tau .
\end{aligned}
$$

On the finite time space-time cylinder $Q_{T}$, analogously to (2.1)-(2.2), we can define

$$
\mathcal{H}^{(s)}\left(Q_{T}\right)=\mathcal{H}^{(s)}\left(\mathbb{R}^{d+1}\right) / \mathcal{H}_{Q_{T}^{\prime}}^{(s)}\left(\mathbb{R}^{d+1}\right)
$$

and

$$
\|u\|_{\mathcal{H}^{(s)}\left(Q_{T}\right)}=\inf _{E}\|E u\|_{\mathcal{H}^{(s)}\left(\mathbb{R}^{d+1}\right)} .
$$

The space $\mathcal{V}^{(s)}\left(Q_{T}\right)$ of solenoidal vector fields is defined as follows:

$$
\mathcal{V}^{(s)}\left(Q_{T}\right)=\left\{\mathbf{v}=\left(v_{1}, \cdots, v_{d}\right) \in\left[H^{(s)}\left(Q_{T}\right)\right]^{d}: \operatorname{div} \mathbf{v}=0\right\} .
$$

The traces of functions $\mathbf{u} \in \mathcal{V}^{(s)}\left(Q_{T}\right)$ on the lateral surface $\Sigma_{T}=(0, T) \times \partial \Omega$ will be sought in certain spaces of the type $H^{s}\left(0, T ; \mathbf{H}^{r}(\partial \Omega)\right)$; their norms are constructed from the norms of $H^{s}\left(\mathbb{R} ; \mathbf{H}^{r}(\partial \Omega)\right)$ by a formula analogous to (2.2). The norm of $H^{s}\left(\mathbb{R} ; \mathbf{H}^{r}(\partial \Omega)\right)$ is defined as follows:

$$
\|\mathbf{u}\|_{H^{s}\left(\mathbb{R} ; \mathbf{H}^{r}(\partial \Omega)\right)}^{2}=\int_{\mathbb{R}}\left(1+|\tau|^{2}\right)^{s}\|\widehat{\mathbf{u}}(\tau, \cdot)\|_{\mathbf{H}^{r}(\partial \Omega)}^{2} d \tau .
$$


Here,

$$
\widehat{\mathbf{u}}(\tau, \cdot)=(2 \pi)^{-1 / 2} \int_{\mathbb{R}} e^{-i \tau t} \mathbf{u}(t, \cdot) d t
$$

is the Fourier transform of the function $\mathbf{u}(t, \cdot)$ defined for $t \in \mathbb{R}$ with values belonging to the space $\mathbf{H}^{r}(\partial \Omega)$. If $s=0$ or $r=0$, we will write

$$
H^{0}\left(0, T ; \mathbf{H}^{r}(\partial \Omega)\right)=L^{2}\left(0, T ; \mathbf{H}^{r}(\partial \Omega)\right)
$$

and

$$
H^{s}\left(0, T ; \mathbf{H}^{0}(\partial \Omega)\right)=H^{s}\left(0, T ; \mathbf{L}^{2}(\partial \Omega)\right) .
$$

Finally, we introduce special logarithmic spaces. Let $B\left(\mathbb{R}^{d}\right)$ be a space of functions $u\left(t, \mathbf{x}^{\prime}\right)$ for $\left(t, \mathbf{x}^{\prime}\right) \in \mathbb{R} \times \mathbb{R}^{d-1}$ with the norm

$$
\|u\|_{B\left(\mathbb{R}^{d}\right)}^{2}=\int_{\mathbb{R}^{d}} b\left(\tau, \boldsymbol{\xi}^{\prime}\right)\left|\widehat{u}\left(\tau, \boldsymbol{\xi}^{\prime}\right)\right|^{2} d \tau d \boldsymbol{\xi}^{\prime}
$$

where $b\left(\tau, \boldsymbol{\xi}^{\prime}\right) \geq 1$ is a weight and $\widehat{u}\left(\tau, \boldsymbol{\xi}^{\prime}\right)$ is the Fourier transform of $u$. Then, by $B_{\ln }(Q)$, where $Q=\mathbb{R} \times \mathbb{R}^{d-1}$, we denote the function space

$$
B_{\ln }(Q)=\text { the completion of } B\left(\mathbb{R}^{d}\right) \text { under the norm }\|u\|_{B_{\ln }(Q)},
$$

where the norm is defined by

$$
\|u\|_{B_{\ln }(Q)}^{2}=\int_{\mathbb{R}^{d}} \frac{b\left(\tau, \boldsymbol{\xi}^{\prime}\right)}{\ln \left[2+\frac{\left(1+|\tau|^{2}\right)}{\left(1+\left|\boldsymbol{\xi}^{\mid}\right|^{2}\right)^{2}}\right]}\left|\widehat{u}\left(\tau, \boldsymbol{\xi}^{\prime}\right)\right|^{2} d \tau d \boldsymbol{\xi}^{\prime} .
$$

For example, $\mathcal{H}_{\ln }^{(s)}\left(\mathbb{R}^{d}\right)$ has the norm

$$
\begin{aligned}
& \|u\|_{\mathcal{H}_{\ln }^{(s)}\left(\mathbb{R}^{d}\right)}^{2} \\
& \quad=\int_{\mathbb{R}^{d}} \frac{\left(1+\left|\boldsymbol{\xi}^{\prime}\right|^{2}\right)^{s}+\left(1+|\tau|^{2}\right)\left(1+\left|\boldsymbol{\xi}^{\prime}\right|^{2}\right)^{s-2}}{\ln \left[2+\left(1+|\tau|^{2}\right) /\left(1+\left|\boldsymbol{\xi}^{\prime}\right|^{2}\right)^{2}\right]}\left|\widehat{u}\left(\tau, \boldsymbol{\xi}^{\prime}\right)\right|^{2} d \boldsymbol{\xi}^{\prime} d \tau
\end{aligned}
$$

as $\mathcal{H}^{(s)}\left(\mathbb{R}^{d}\right)$ has the norm (2.4). The space $H_{\ln }^{s}\left(\mathbb{R} ; H^{r}\left(\mathbb{R}^{d-1}\right)\right)$ has the norm

$$
\begin{aligned}
& \|u\|_{H_{\ln }^{s}\left(\mathbb{R} ; H^{r}\left(\mathbb{R}^{d-1}\right)\right)}^{2} \\
& \quad=\int_{\mathbb{R}^{d}} \frac{\left(1+\left|\boldsymbol{\xi}^{\prime}\right|^{2}\right)^{r}\left(1+|\tau|^{2}\right)^{s}}{\ln \left[2+\left(1+|\tau|^{2}\right) /\left(1+\left|\boldsymbol{\xi}^{\prime}\right|^{2}\right)^{2}\right]}\left|\widehat{u}\left(\tau, \boldsymbol{\xi}^{\prime}\right)\right|^{2} d \boldsymbol{\xi}^{\prime} d \tau .
\end{aligned}
$$

If $B(\Sigma)$ is a function space defined on $\Sigma=\mathbb{R} \times \partial \Omega$, then the logarithmic space $B_{\ln }(\Sigma)$ is constructed from $B_{\ln }\left(\mathbb{R}^{d}\right)$ in the usual manner just as $B(\Sigma)$ is constructed from $B\left(\mathbb{R}^{d}\right)$; see above.

2.2. Formulation of the trace theorem. As is well known, a trace theorem consists of two parts: a restriction theorem and an extension theorem. Thus, below, we formulate the desired trace theorem as two separate assertions.

Let $\mathbf{v}(\mathbf{x})$, defined for $\mathbf{x} \in \Omega$, be a solenoidal vector field of class $C^{\infty}$, and $\gamma \mathbf{v}$ its restriction on the boundary $\partial \Omega$ :

$$
\gamma \mathbf{v}=\left.\mathbf{v}\right|_{\partial \Omega}
$$

We can decompose the vector field $\gamma \mathbf{v}$ into the tangential component $\gamma_{\tau} \mathbf{v}$ and the normal component $\left(\gamma_{n} \mathbf{v}\right) \mathbf{n}$ :

$$
\gamma \mathbf{v}=\gamma_{\tau} \mathbf{v}+\left(\gamma_{n} \mathbf{v}\right) \mathbf{n}
$$


Here, $\gamma_{n} \mathbf{v}$ is the orthogonal projection of $\gamma \mathbf{v}$ onto the normal vector field $\mathbf{n}$, and $\gamma_{\tau} \mathbf{v}$ is the orthogonal projection of $\gamma \mathbf{v}$ into the tangent space $T_{\mathbf{x}} \partial \Omega$ to the manifold $\partial \Omega$ at the point $\mathbf{x}$. We will also use the decomposition (2.9) for the restriction $\gamma \mathbf{v}$ to the lateral boundary $\Sigma_{T}$ when $\mathbf{v}(t, \mathbf{x})$ is defined on $Q_{T}$; the meaning of the decomposition in this case is evident.

The case $d \equiv \operatorname{dim} \Omega=3$ is the most important case for applications, and we will consider only this case in the sequel.

To formulate the restriction and extension theorems in a compact form, we introduce the space of traces. Specifically we introduce the function spaces on $\Sigma_{T}$ :

$$
G_{\tau}^{s}\left(\Sigma_{T}\right)= \begin{cases}{\left[\mathcal{H}^{(s-1 / 2)}\left(\Sigma_{T}\right)\right]^{2},} & s \geq 5 / 2 \\ {\left[L^{2}\left(0, T ; H^{s-1 / 2}(\partial \Omega)\right)\right.} & 2 \leq s \leq 5 / 2 \\ \left.\cap H^{(2 s-1) / 4}\left(0, T ; L^{2}(\partial \Omega)\right)\right]^{2}, & \\ {\left[L^{2}\left(0, T ; H^{s-1 / 2}(\partial \Omega)\right)\right.} & 1 / 2<s \leq 2 \\ \left.\cap H^{\frac{2 s-1}{2 s}}\left(0, T ; H^{\left(1-\frac{2}{s}\right)\left(s-\frac{1}{2}\right)}(\partial \Omega)\right)\right]^{2}, & \end{cases}
$$

and

$$
G_{n}^{s}\left(\Sigma_{T}\right)=\left\{\begin{aligned}
\mathcal{H}^{(s-1 / 2)}\left(\Sigma_{T}\right) \cap L^{2}\left(0, T ; \widetilde{H}^{1}(\partial \Omega)\right), & s \geq 3 / 2, \\
L^{2}\left(0, T ; \widetilde{H}^{s-1 / 2}(\partial \Omega)\right) & 1 \leq s \leq 3 / 2, \\
\cap H^{(2 s+1) / 4}\left(0, T ; \widetilde{H}^{-1}(\partial \Omega)\right), & \\
L^{2}\left(0, T ; \widetilde{H}^{s-1 / 2}(\partial \Omega)\right) & \text { for } 1 / 2<s \leq 1, \\
\cap H^{\frac{2 s+1}{2 s+2}}\left(0, T ; \widetilde{H}^{\frac{2 s^{2}-3 s-3}{2 s+2}}(\partial \Omega)\right), &
\end{aligned}\right.
$$

where $\widetilde{H}^{\alpha}(\partial \Omega)=\left\{v \in H^{\alpha}(\partial \Omega): \int_{\partial \Omega} v d \mathbf{x}=0\right\}$ and, for $\alpha<0$, the integral is understood in the sense of distributions. We set

$$
G^{s}\left(\Sigma_{T}\right)=G_{\tau}^{s}\left(\Sigma_{T}\right) \times G_{n}^{s}\left(\Sigma_{T}\right)
$$

Theorem 2.1 (The restriction theorem). Let $\Omega \subset \mathbb{R}^{3}$. Then, the operator (2.9) can be extended by continuity into the following continuous operator:

$$
\gamma: \mathcal{V}^{(s)}\left(Q_{T}\right) \rightarrow G^{s}\left(\Sigma_{T}\right) \quad \text { for } s>1 / 2, s \neq 3 / 2, s \neq 5 / 2 .
$$

If $s=5 / 2$, then the restriction operator

$$
\gamma=\left(\gamma_{\tau}, \gamma_{n}\right): \mathcal{V}^{(5 / 2)}\left(Q_{T}\right) \rightarrow\left[\mathcal{H}_{\ln }^{(2)}\left(\Sigma_{T}\right)\right]^{2} \times \mathcal{H}^{(2)}\left(\Sigma_{T}\right)
$$

is continuous. For $s=3 / 2$, the restriction operator $\gamma=\left(\gamma_{\tau}, \gamma_{n}\right)$ as a mapping

$$
\begin{aligned}
& \gamma: \mathcal{V}^{(3 / 2)}\left(Q_{T}\right) \rightarrow\left[L^{2}\left(0, T ; H^{1}(\partial \Omega)\right) \cap H^{2 / 3}\left(0, T ; H^{-1 / 3}(\partial \Omega)\right)\right]^{2} \\
& \quad \times\left[L^{2}\left(0, T ; \widetilde{H}^{1}(\partial \Omega)\right) \cap H_{\ln }^{1}\left(0, T ; \widetilde{H}^{-1}(\partial \Omega)\right) \cap H^{2 / 3}\left(0, T ; H^{-1 / 3}(\partial \Omega)\right)\right]
\end{aligned}
$$

is bounded, where the logarithmic spaces are defined in (2.7) and (2.8).

Theorem 2.2 (The extension theorem). Let $\Omega \subset \mathbb{R}^{3}$. Then for $s>1 / 2$ there exists a continuous extension operator

$$
R: G^{s}\left(\Sigma_{T}\right) \rightarrow \mathcal{V}^{(s)}\left(Q_{T}\right),
$$

i.e., the operator $R$ is such that $\gamma \circ R=I$, where $I: G^{s}\left(\Sigma_{T}\right) \rightarrow G^{s}\left(\Sigma_{T}\right)$ is the identity operator. 
Remark 2.1. Theorems 2.1 and 2.2 imply that the restriction operator $(2.13)$ is surjective for $s>1 / 2, s \neq 3 / 2$ and $s \neq 5 / 2$. If $s=3 / 2$, then $G^{3 / 2}\left(\Sigma_{T}\right) \subset \Im(\gamma)$, where $\Im(\gamma)$ is the image of the operator (2.15). Analogously, if $s=5 / 2$, then $G^{5 / 2}\left(\Sigma_{T}\right) \subset \Im(\gamma)$, where $\Im(\gamma)$ is the image of the operator (2.14).

Sections 3, 4, and 5 are devoted to the proofs of Theorems 2.1 and 2.2, which are the main results of this paper. We also prove some other extension results.

\section{Trace theorems for the Sobolev spaces $\mathcal{H}^{(s)}\left(\mathbb{R}^{d+1}\right)$}

3.1. Restriction results. For a scalar function $u(t, \mathbf{x}) \in C^{\infty}\left(\mathbb{R}^{d+1}\right) \cap \mathcal{H}^{(s)}\left(\mathbb{R}^{d+1}\right)$, we introduce the following restriction operators:

$$
\left(\gamma_{0} u\right)\left(t, \mathbf{x}^{\prime}\right)=\left.u(t, \mathbf{x})\right|_{x_{d}=0} \quad \text { and } \quad\left(\gamma_{k} u\right)\left(t, \mathbf{x}^{\prime}\right)=\left.\frac{\partial^{k} u(t, \mathbf{x})}{\partial x_{d}^{k}}\right|_{x_{d}=0}
$$

where $k=1,2, \ldots, \mathbf{x}=\left(x_{1}, \ldots, x_{d}\right)$, and $\mathbf{x}^{\prime}=\left(x_{1}, \ldots, x_{d-1}\right)$. Our aim is to determine the spaces of restrictions $\gamma_{k} u(k=0,1,2, \ldots)$ when $u \in \mathcal{H}^{(s)}\left(\mathbb{R}^{d+1}\right)$. We will assume that

$$
s-k>1 / 2 .
$$

First of all, we consider the case

$$
1 / 2<s-k<5 / 2 .
$$

Lemma 3.1. Let $k \geq 0$ be an integer and assume that the inequality (3.2) holds. Then, the operator $\gamma_{k}$ defined in (3.1) can be extended by continuity into the continuous operator

$$
\begin{aligned}
\gamma_{k}: \mathcal{H}^{(s)}\left(\mathbb{R}^{d+1}\right) \rightarrow & L^{2}\left(\mathbb{R} ; H^{s-k-1 / 2}\left(\mathbb{R}^{d-1}\right)\right) \\
& \cap H^{(2 s-2 k-1) / 4}\left(\mathbb{R} ; L^{2}\left(\mathbb{R}^{d-1}\right)\right), \quad \text { if } s \geq 2,
\end{aligned}
$$

and

$$
\begin{aligned}
\gamma_{k} & : \mathcal{H}^{(s)}\left(\mathbb{R}^{d+1}\right) \rightarrow L^{2}\left(\mathbb{R} ; H^{s-k-1 / 2}\left(\mathbb{R}^{d-1}\right)\right) \\
& \cap H^{(2 s-2 k-1) /(2 s)}\left(\mathbb{R} ; H^{(1-2 / s)(s-k-1 / 2)}\left(\mathbb{R}^{d-1}\right)\right), \quad \text { if } s \in(1 / 2,2) .
\end{aligned}
$$

Proof. Let $\widehat{u}(\tau, \boldsymbol{\xi})$ denote the Fourier transform of $u(t, \mathbf{x})$; then

$$
\begin{aligned}
\widehat{\gamma_{k} u}\left(\tau, \boldsymbol{\xi}^{\prime}\right) & =\left.(2 \pi)^{-1 / 2} \frac{\partial^{k}}{\partial x_{d}^{k}} \int_{\mathbb{R}} e^{i x_{d} \xi_{d}} \widehat{u}\left(\tau, \boldsymbol{\xi}^{\prime}, \xi_{d}\right) d \xi_{d}\right|_{x_{d}=0} \\
& =(2 \pi)^{-1 / 2} \int_{\mathbb{R}}\left(i \xi_{d}\right)^{k} \widehat{u}\left(\tau, \boldsymbol{\xi}^{\prime}, \xi_{d}\right) d \xi_{d} .
\end{aligned}
$$

Let

$$
a\left(\boldsymbol{\xi}^{\prime}\right)=1+\left|\boldsymbol{\xi}^{\prime}\right|^{2}, \quad b(\tau)=1+|\tau|^{2},
$$

and

$$
\Lambda_{s}(\tau, \boldsymbol{\xi})=a^{s}\left(\boldsymbol{\xi}^{\prime}\right)+\left|\xi_{d}\right|^{2 s}+b(\tau)\left[a^{s-2}\left(\boldsymbol{\xi}^{\prime}\right)+\left|\xi_{d}\right|^{2(s-2)}\right]
$$


Applying the Plancherel theorem and the Cauchy-Schwarz-Bunyakovsky inequality to $(3.5)$, we obtain, with a function $R\left(\tau, \boldsymbol{\xi}^{\prime}\right)>0$,

$$
\begin{gathered}
\int_{\mathbb{R}^{d}} R\left(\tau, \boldsymbol{\xi}^{\prime}\right)\left|\widehat{\gamma_{k} u}\left(\tau, \boldsymbol{\xi}^{\prime}\right)\right|^{2} d \tau d \boldsymbol{\xi}^{\prime} \\
=\int_{\mathbb{R}^{d}} R\left(\tau, \boldsymbol{\xi}^{\prime}\right)\left|\int_{\mathbb{R}} \frac{\xi_{d}^{k}}{\Lambda_{s}^{1 / 2}(\tau, \boldsymbol{\xi})} \widehat{u}\left(\tau, \boldsymbol{\xi}^{\prime}, \xi_{d}\right) \Lambda_{s}^{1 / 2}(\tau, \boldsymbol{\xi}) d \xi_{d}\right|^{2} d \tau d \boldsymbol{\xi}^{\prime} \\
\leq C \int_{\mathbb{R}^{d}}\left(R\left(\tau, \boldsymbol{\xi}^{\prime}\right) \int_{\mathbb{R}} \frac{\eta^{2 k}}{\Lambda_{s}\left(\tau, \boldsymbol{\xi}^{\prime}, \eta\right)} d \eta\right) \\
\cdot \int_{\mathbb{R}} \Lambda_{s}(\tau, \boldsymbol{\xi})\left|\widehat{u}\left(\tau, \boldsymbol{\xi}^{\prime}, \xi_{d}\right)\right|^{2} d \xi_{d} d \tau d \boldsymbol{\xi}^{\prime} .
\end{gathered}
$$

If we could find $R\left(\tau, \boldsymbol{\xi}^{\prime}\right)$ such that

$$
0<C_{1} \leq\left|R\left(\tau, \boldsymbol{\xi}^{\prime}\right) \int_{\mathbb{R}} \frac{\eta^{2 k}}{\Lambda_{s}\left(\tau, \boldsymbol{\xi}^{\prime}, \eta\right)} d \eta\right| \leq C_{2},
$$

where the constants $C_{1}$ and $C_{2}$ do not depend on $\tau$ and $\boldsymbol{\xi}^{\prime}$, then (3.8) would imply

$$
\int_{\mathbb{R}^{d}} R\left(\tau, \boldsymbol{\xi}^{\prime}\right)\left|\widehat{\gamma_{k} u}\left(\tau, \boldsymbol{\xi}^{\prime}\right)\right|^{2} d \tau d \boldsymbol{\xi}^{\prime} \leq C\|u\|_{\mathcal{H}^{(s)}\left(\mathbb{R}^{d+1}\right)}^{2},
$$

so that the function $R$ would define a norm on the space for $\gamma_{k} u, u \in \mathcal{H}^{(s)}\left(\mathbb{R}^{d+1}\right)$.

Let us determine an $R$ satisfying (3.9). Set

$$
\beta=a^{s}+b a^{s-2} .
$$

Using this last relation and (3.6)-(3.7), we obtain, by making some simple transformations,

$$
\begin{aligned}
\int_{\mathbb{R}} & \frac{\eta^{2 k}}{\Lambda_{s}\left(\tau, \boldsymbol{\xi}^{\prime}, \eta\right)} d \eta=2 \int_{0}^{\infty} \frac{\eta^{2 k}}{a^{s}+b a^{s-2}+\eta^{2 s}+b \eta^{2(s-2)}} d \eta \\
= & \frac{2}{\beta^{1-(2 k+1) /(2 s)}} \\
& \cdot \int_{0}^{\infty} \frac{\left(\eta / \beta^{1 /(2 s)}\right)^{2 k}}{1+\left(\eta / \beta^{1 /(2 s)}\right)^{2 s}+\left(b / \beta^{2 / s}\right)\left(\eta / \beta^{1 /(2 s)}\right)^{2(s-2)}} d \eta / \beta^{1 /(2 s)} \\
= & 2\left(a^{s}+b a^{s-2}\right)^{(2 k+1-2 s) /(2 s)} \int_{0}^{\infty} \frac{y^{2 k}}{1+y^{2 s}+\frac{b}{\beta^{2 / s}} y^{2(s-2)}} d y
\end{aligned}
$$

Thus, we have to estimate the integral

$$
I(r)=\int_{0}^{\infty} \frac{y^{2 k}}{1+y^{2 s}+r y^{2(s-2)}} d y,
$$

where

$$
r=\frac{b}{\beta^{2 / s}}=\frac{b}{\left(a^{s}+b a^{s-2}\right)^{2 / s}} .
$$


Through the change of variable $z=y^{2 k+1}$ we obtain, when $s-k$ satisfies (3.2),

$$
\begin{aligned}
I(r) & =\frac{1}{2 k+1} \int_{0}^{\infty} \frac{d z}{1+z^{2 s /(2 k+1)}+r z^{2(s-2) /(2 k+1)}} \\
& \leq \frac{1}{2 k+1} \int_{0}^{\infty} \frac{d z}{z^{2(s-2) /(2 k+1)}\left(z^{4 /(2 k+1)}+r\right)} \\
& =\frac{1}{2 k+1}\left(1-\frac{2(s-2)}{2 k+1}\right)^{-1} \int_{0}^{\infty} \frac{d z^{(2 k+5-2 s) /(2 k+1)}}{z^{4 /(2 k+1)}+r} \\
& =\frac{1}{2 k+5-2 s} \int_{0}^{\infty} \frac{d y}{y^{4 /(2 k+5-2 s)}+r} \\
& =C r^{(2 k+1-2 s) / 4} \int_{0}^{\infty} \frac{d y / r^{(2 k+5-2 s) / 4}}{\left(y / r^{(2 k+5-2 s) / 4}\right)^{4 /(2 k+5-2 s)}+1} \\
& =C r^{(2 k+1-2 s) / 4} .
\end{aligned}
$$

On the other hand, analogously to (3.13), we have

$$
\begin{aligned}
(2 k & +1) I(r)>\int_{1}^{\infty} \frac{d z}{2 z^{2(s-2) /(2 k+1)}\left(z^{4 /(2 k+1)}+r\right)} \\
& =C r^{(2 k+1-2 s) / 4} \int_{1}^{\infty} \frac{d y / r^{(2 k+5-2 s) / 4}}{\left(y / r^{(2 k+5-2 s) / 4}\right)^{4 /(2 k+5-2 s)}+1} \\
& \geq C_{1} r^{(2 k+1-2 s) / 4} \text { as } r \rightarrow \infty .
\end{aligned}
$$

Relations (3.13)-(3.14) and the evident relation $I(r) \rightarrow$ constant as $r \rightarrow 0$ yield

$$
I(r) \sim(r+1)^{(2 k+1-2 s) / 4} \text { for } r \geq 0,
$$

i.e., $c_{1}(r+1)^{(2 k+1-2 s) / 4} \leq I(r) \leq c_{2}(r+1)^{(2 k+1-2 s) / 4}$. Substituting the expression for $r$ defined in (3.12) into (3.15) and taking into account (3.9) and (3.10), we obtain

$$
\begin{aligned}
& R\left(\tau, \boldsymbol{\xi}^{\prime}\right) \sim\left(a^{s}+b a^{s-2}\right)^{-\frac{2 k+1-2 s}{2 s}} \cdot\left(1+\frac{b}{\left(a^{s}+b a^{s-2}\right)^{2 / s}}\right)^{-(2 k+1-2 s) / 4} \\
& \sim\left(a^{s}+b a^{s-2}\right)^{-\frac{2 k+1-2 s}{2 s}+\frac{2 k+1-2 s}{2 s}} \cdot\left(a^{2}+b^{2 / s} a^{2(s-2) / s}+b\right)^{-(2 k+1-2 s) / 4} \\
& \sim\left(a^{2}+b\right)^{(2 s-2 k-1) / 4}, \quad \text { if } s \geq 2,
\end{aligned}
$$

where the condition $s \geq 2$ was used only in the last step of (3.16). By the definition (3.6) of the functions $a\left(\boldsymbol{\xi}^{\prime}\right)$ and $b(\tau)$ and the definition (2.6) of the space $H^{s}\left(\mathbb{R} ; H^{r}\left(\mathbb{R}^{d-1}\right)\right)$, we see that $R\left(\tau, \boldsymbol{\xi}^{\prime}\right)$ in (3.16) defines a norm for the space

$$
L^{2}\left(\mathbb{R} ; H^{s-k-1 / 2}\left(\mathbb{R}^{d-1}\right)\right) \cap H^{(2 s-2 k-1) / 4}\left(\mathbb{R} ; L^{2}\left(\mathbb{R}^{d-1}\right)\right), \quad s \geq 2 .
$$


Analogously, when $1 / 2<s \leq 2$ we obtain

$$
\begin{aligned}
& R\left(\tau, \boldsymbol{\xi}^{\prime}\right) \sim\left(a^{2}+b^{2 / s} a^{2(s-2) / s}+b\right)^{(2 s-2 k-1) / 4} \\
& \sim\left(a^{2(s-2) / s}\left(a^{4 / s}+b^{2 / s}+b a^{2(2-s) / s}\right)\right)^{(2 s-2 k-1) / 4} \\
& \sim\left(a^{2(s-2) / s}\left(a^{4 / s}+b^{2 / s}\right)\right)^{(2 s-2 k-1) / 4} \\
& \sim a^{s-k-1 / 2}+b^{(s-k-1 / 2) / s} a^{(s-2)(s-k-1 / 2) / s}
\end{aligned}
$$

and we see that $R\left(\tau, \boldsymbol{\xi}^{\prime}\right)$ defines a norm for the space

$$
L^{2}\left(\mathbb{R} ; H^{s-k-1 / 2}\left(\mathbb{R}^{d-1}\right)\right) \cap H^{(2 s-2 k-1) /(2 s)}\left(\mathbb{R} ; H^{(1-2 / s)(s-k-1 / 2)}\left(\mathbb{R}^{d-1}\right)\right)
$$

for $1 / 2<s \leq 2$.

Next we consider the case

$$
s-k=5 / 2 .
$$

Lemma 3.2. Let $k \geq 0$ be an integer and assume (3.17) holds. Then, the operator $\gamma_{k}$ defined in (3.1) can be extended by continuity into the continuous operator

$$
\gamma_{k}: \mathcal{H}^{(5 / 2)}\left(\mathbb{R}^{d+1}\right) \rightarrow \mathcal{H}_{\mathrm{ln}}^{(2)}\left(\mathbb{R}^{d}\right)
$$

Proof. As in the previous lemma, we have to estimate the integral $I(r)$ from $(3.11)$ when $s-k$ satisfies (3.17). By the first equality in (3.13), we have

$$
(2 k+1) I(r)=\int_{0}^{1} \frac{d z}{1+z^{\frac{2 k+5}{2 k+1}}+r z}+\int_{1}^{\infty} \frac{d z}{1+z^{\frac{2 k+5}{2 k+1}}+r z} .
$$

Through a change of variables, we have

$$
\int_{0}^{1} \frac{d z}{1+z^{\frac{2 k+5}{2 k+1}}+r z} \leq \int_{0}^{1} \frac{d z}{1+r z}=\frac{1}{r} \int_{0}^{r} \frac{d y}{1+y}=\frac{\ln (1+r)}{r}
$$

and

$$
\int_{0}^{1} \frac{d z}{1+z^{\frac{2 k+5}{2 k+1}}+r z} \geq \int_{0}^{1} \frac{d z}{2+r z}=\frac{1}{r} \int_{0}^{r} \frac{d y}{2+y}=\frac{\ln (2+r)-\ln 2}{r} .
$$

On the other hand,

$$
\begin{aligned}
\int_{1}^{\infty} & \frac{d z}{1+z^{\frac{2 k+5}{2 k+1}}+r z} \leq \int_{1}^{\infty} \frac{d z}{z^{\frac{2 k+5}{2 k+1}}\left(1+r z^{1-\frac{2 k+5}{2 k+1}}\right)} \\
\quad= & \left(1-\frac{2 k+5}{2 k+1}\right)^{-1} \int_{1}^{\infty} \frac{d z^{-4 /(2 k+1)}}{1+r z^{-4 /(2 k+1)}}=\frac{2 k+1}{4} \int_{0}^{1} \frac{d y}{1+r y} \\
\quad= & \frac{2 k+1}{4} \frac{\ln (1+r)}{r}
\end{aligned}
$$

If $r \geq 1$, then

$$
\begin{gathered}
\int_{1}^{\infty} \frac{d z}{1+z^{\frac{2 k+5}{2 k+1}}+r z} \geq \int_{1}^{\infty} \frac{d z}{z^{\frac{2 k+5}{2 k+1}}\left(1+2 r z^{1-\frac{2 k+5}{2 k+1}}\right)} \\
\quad=\frac{2 k+1}{4} \int_{0}^{1} \frac{d y}{1+2 r y}=\frac{2 k+1}{4} \frac{\ln (1+2 r)}{2 r} .
\end{gathered}
$$


Also, as $r \rightarrow 0$, we have

$$
\int_{0}^{\infty} \frac{d z}{1+z^{\frac{2 k+5}{2 k+1}}+r z} \sim 1
$$

These relations imply

$$
I(r) \sim \frac{\ln (r+2)}{1+r} \quad \text { for } r \in(0, \infty) .
$$

With the help of (3.19), and denoting $\beta=\left(a^{k+5 / 2}+b a^{k+1 / 2}\right)^{4 /(2 k+5)}$ (analogously to $(3.16))$, we may deduce

$$
\begin{aligned}
& R\left(\tau, \boldsymbol{\xi}^{\prime}\right) \sim \beta\left(1+\frac{b}{\beta}\right) / \ln \left(2+\frac{b}{\beta}\right) \sim(\beta+b) / \ln \left(2+\frac{b}{\beta}\right) \\
& \sim\left(a^{2}+b^{4 /(2 k+5)} a^{2(2 k+1) /(2 k+5)}+b\right) / \\
& \quad \ln \left(\frac{2\left(a^{2}+b^{4 /(2 k+5)} a^{2(2 k+1) /(2 k+5)}\right)+b}{a^{2}+b^{4 /(2 k+5)} a^{2(2 k+1) /(2 k+5)}}\right) \\
& \sim\left(a^{2}+b\right) / \ln \left(\frac{a^{2}+b}{a^{2(2 k+1) /(2 k+5)}\left(a^{8 /(2 k+5)}+b^{4 /(2 k+5)}\right)}\right) \\
& \sim \frac{a^{2}+b}{\ln \left(\frac{a^{2}+b}{a^{2}}\right)^{(2 k+1) /(2 k+5)}} \sim \frac{a^{2}+b}{\ln \left(1+\frac{b}{a^{2}}\right)} .
\end{aligned}
$$

This relation and (3.8) yield (3.18).

Finally, we consider the case

$$
s-k>5 / 2 \text {. }
$$

Lemma 3.3. Let $k \geq 0$ be an integer and assume that (3.20) holds. Then, the operator $\gamma_{k}$ defined in (3.1) can be extended by continuity into the continuous operator

$$
\gamma_{k}: \mathcal{H}^{(s)}\left(\mathbb{R}^{d+1}\right) \rightarrow \mathcal{H}^{(s-k-1 / 2)}\left(\mathbb{R}^{d}\right)
$$

Proof. We have to estimate $I(r)$ from (3.11) under condition (3.20), which is equivalent to the inequality $2(s-2) /(2 k+1)>1$. Using the first equality from (3.13), we obtain

$$
\begin{aligned}
& (2 k+1) I(r) \leq \int_{0}^{\infty} \frac{d z}{1+r z^{2(s-2) /(2 k+1)}} \\
& \quad=r^{-\frac{2 k+1}{2(s-2)}} \int_{0}^{\infty} \frac{d z r^{\frac{2 k+1}{2(s-2)}}}{1+\left(z r^{\frac{2 k+1}{2(s-2)}}\right)^{2(s-2) /(2 k+1)}}=C r^{-\frac{2 k+1}{2(s-2)}} .
\end{aligned}
$$

Since $z^{2 s /(2 k+1)}<r z^{2(s-2) /(2 k+1)}$ when $z<r^{(2 k+1) / 4}$, we have

$$
\begin{aligned}
(2 k+ & 1) I(r) \geq \int_{0}^{r^{(2 k+1) / 4}} \frac{d z}{1+2 r z^{2(s-2) /(2 k+1)}} \\
& =r^{-\frac{2 k+1}{2(s-2)}} \int_{0}^{r^{(2 k+1) / 4}} \frac{d z r^{\frac{2 k+1}{2(s-2)}}}{1+2\left(z r^{\frac{2 k+1}{2(s-2)}}\right)^{2(s-2) /(2 k+1)}} \\
& =r^{-\frac{2 k+1}{2(s-2)}} \int_{0}^{r^{(2 k+1) / 4+(2 k+1) / 2(s-2)}} \frac{d y}{1+2 y^{\frac{2(s-2)}{2 k+1}}} \\
& \geq C r^{-\frac{2 k+1}{2(s-2)}} \text { as } r \rightarrow \infty .
\end{aligned}
$$


The first equality in (3.13) yields that

$$
I(r) \sim \text { constant } \quad \text { as } r \rightarrow 0 .
$$

Hence, by (3.22)-(3.23), we obtain

$$
I(r) \sim(1+r)^{-\frac{2 k+1}{2(s-2)}} \quad \text { for } r \geq 0 .
$$

Using this relation we obtain, similarly to (3.16),

$$
\begin{aligned}
& R\left(\tau, \boldsymbol{\xi}^{\prime}\right) \sim\left(a^{s}+b a^{s-2}\right)^{-\frac{2 k+1-2 s}{2 s}} \cdot\left(1+\frac{b}{\left(a^{s}+b a^{s-2}\right)^{2 / s}}\right)^{\frac{2 k+1}{2(s-2)}} \\
& \sim\left(a^{s}+b a^{s-2}\right)^{1-\frac{2 k+1}{2 s}-\frac{2 k+1}{s(s-2)}} \\
& \cdot\left(a^{2}+b^{2 / s} a^{2(s-2) / s}+b\right)^{\frac{2 k+1}{2(s-2)}} \\
& \sim a^{(s-2)\left(1-\frac{2 k+1}{2(s-2)}\right)}\left(a^{2}+b\right) \sim a^{s-k-5 / 2}\left(a^{2}+b\right) .
\end{aligned}
$$

Taking into account (3.6) and (2.4), we see that $R\left(\tau, \boldsymbol{\xi}^{\prime}\right)$ in (3.24) defines a norm of the space $\mathcal{H}^{(s-k-1 / 2)}\left(\mathbb{R}^{d}\right)$.

Consider now the finite time case $Q_{T}=(0, T) \times \Omega$ and $\Sigma_{T}=(0, T) \times \partial \Omega$. For a scalar function $u(t, \mathbf{x}) \in C^{\infty}\left(\overline{Q_{T}}\right) \cap \mathcal{H}^{(s)}\left(Q_{T}\right)$, define the restriction operator $\gamma_{k}$ by the formula

$$
\gamma_{0} u=\left.u\right|_{\Sigma_{T}}, \quad \gamma_{k} u=\left.\gamma \frac{\partial^{k} u}{\partial n^{k}} \equiv \frac{\partial^{k} u}{\partial n^{k}}\right|_{\Sigma_{T}}, \quad k=1,2, \ldots
$$

where $\frac{\partial^{k}}{\partial n^{k}}$ is the $k$-th normal derivative. Lemmas $3.1,3.2$, and 3.3 imply the following restriction result for functions defined on $Q_{T}$.

Theorem 3.1. Let $k \geq 0$ be an integer and let $s>0$. Assume $s-k>1 / 2$. Then, the restriction operator $\gamma_{k}$ defined in (3.25) can be extended by continuity into the bounded operators

$$
\begin{aligned}
\gamma_{k}: \mathcal{H}^{(s)}\left(Q_{T}\right) \rightarrow \mathcal{H}^{(s-k-1 / 2)}\left(\Sigma_{T}\right) \quad \text { for } s-k>5 / 2, \\
\gamma_{k}: \mathcal{H}^{(s)}\left(Q_{T}\right) \rightarrow L^{2}\left(0, T ; H^{s-k-1 / 2}(\partial \Omega)\right) \\
\cap H^{(2 s-2 k-1) / 4}\left(0, T ; L^{2}(\partial \Omega)\right) \quad \text { for } 1 / 2<s-k<5 / 2, s \geq 2, \\
\gamma_{k}: \mathcal{H}^{(s)}\left(Q_{T}\right) \rightarrow L^{2}\left(0, T ; H^{s-k-1 / 2}(\partial \Omega)\right) \\
\cap H^{(2 s-2 k-1) /(2 s)}\left(0, T ; H^{(1-2 / s)(s-k-1 / 2)}(\partial \Omega)\right) \\
\quad \text { for } 1 / 2<s-k<5 / 2,1 / 2<s<2,
\end{aligned}
$$

and

$$
\gamma_{k}: \mathcal{H}^{(5 / 2)}\left(Q_{T}\right) \rightarrow \mathcal{H}_{\ln }^{(2)}\left(\Sigma_{T}\right) \quad \text { for } s-k=5 / 2
$$

where $\mathcal{H}_{\ln }^{(2)}\left(\Sigma_{T}\right)$ is constructed in the usual manner from the space $\mathcal{H}_{\ln }^{(2)}\left(\mathbb{R}^{d}\right)$ defined through (2.7).

Theorem 3.1 can be deduced from Lemmas 3.1-3.3 by standard methods that involve the extension of $u \in \mathcal{H}^{(s)}\left(Q_{T}\right)$ to $u \in \mathcal{H}^{(s)}\left(\mathbb{R}^{d+1}\right)$, the introduction of a partition of unity in a neighborhood of $\Sigma_{T}$, and the rectification of $\Sigma_{T}$. 
3.2. Extension results. We begin with the construction of the extension operators which correspond to the restriction operator (3.21).

Lemma 3.4. Let $k \geq 0$ be an integer and $s \in \mathbb{R}$. Then, there exists a continuous operator

$$
\beta_{k}: \mathcal{H}^{(s-k-1 / 2)}\left(\mathbb{R}^{d}\right) \rightarrow \mathcal{H}^{(s)}\left(\mathbb{R}^{d+1}\right)
$$

such that $\gamma_{k} \circ \beta_{k} u=u$ and $\gamma_{j} \circ \beta_{k} u=0, j \neq k$, for each $u \in \mathcal{H}^{(s-k-1 / 2)}\left(\mathbb{R}^{d}\right)$, where $\gamma_{k}$ is the operator (3.21).

Proof. Let $\phi_{k}(t) \in C^{\infty}(\mathbb{R})$ with $\operatorname{supp} \phi_{k} \subset[-1,1]$ and $\phi_{k}(t)=t^{k} / k$ ! for $|t|<1 / 2$. For $u(t, \mathbf{x}) \in \mathcal{H}^{(s)}\left(\mathbb{R}^{d+1}\right)$ we denote by

$$
\widetilde{u}\left(\tau, \boldsymbol{\xi}^{\prime}, x_{d}\right) \equiv \widetilde{u}\left(\tau, \xi_{1}, \cdots, \xi_{d-1}, x_{d}\right)
$$

the Fourier transform with respect to the variables $\left(t, \mathbf{x}^{\prime}\right)=\left(t, x_{1}, \cdots, x_{d-1}\right)$, i.e., we use $\widetilde{u}\left(\tau, \boldsymbol{\xi}^{\prime}, x_{d}\right)$ to denote the partial Fourier transform (to distinguish it from the total Fourier transform $\widehat{u}$ with respect to all variables). Let $v\left(t, \mathbf{x}^{\prime}\right) \in$ $\mathcal{H}^{(s-k-1 / 2)}\left(\mathbb{R}^{d}\right)$, and let $\widehat{v}\left(\tau, \boldsymbol{\xi}^{\prime}\right)$ be the Fourier transform of $v$. We define the operator $\beta_{k}$ by the formula

$$
\left(\widetilde{\beta_{k} v}\right)\left(\tau, \boldsymbol{\xi}^{\prime}, x_{d}\right)=\phi_{k}\left(a^{1 / 2}\left(\boldsymbol{\xi}^{\prime}\right) x_{d}\right) \widehat{u}\left(\tau, \boldsymbol{\xi}^{\prime}\right) a^{-k / 2}\left(\boldsymbol{\xi}^{\prime}\right),
$$

where the function $a\left(\boldsymbol{\xi}^{\prime}\right)$ was defined in (3.6). Taking the Fourier transform with respect to $x_{d}$ in (3.31), we obtain the complete Fourier transform of $\beta_{k} v$ :

$$
\widehat{\beta_{k} v}(\tau, \boldsymbol{\xi})=\frac{\widehat{u}\left(\tau, \boldsymbol{\xi}^{\prime}\right)}{a^{(1+k) / 2}\left(\boldsymbol{\xi}^{\prime}\right)} \widehat{\phi}_{k}\left(\xi_{d} a^{-1 / 2}\left(\boldsymbol{\xi}^{\prime}\right)\right) .
$$

Taking into account the definition (2.4) of the norm of the space $\mathcal{H}^{(s)}\left(\mathbb{R}^{d+1}\right)$ and (3.32), we obtain

$$
\begin{aligned}
& \left\|\beta_{k} v\right\|_{\mathcal{H}^{(s)}}^{2}\left(\mathbb{R}^{d+1}\right) \\
& =\int\left(\left[a\left(\boldsymbol{\xi}^{\prime}\right)+\xi_{d}^{2}\right]^{s}+b(\tau)\left[a\left(\boldsymbol{\xi}^{\prime}\right)+\xi_{d}^{2}\right]^{s-2}\right) \\
& \quad \cdot \frac{\left|\widehat{u}\left(\tau, \boldsymbol{\xi}^{\prime}\right)\right|^{2}}{a^{(1+k)}\left(\boldsymbol{\xi}^{\prime}\right)}\left|\widehat{\phi}_{k}\left(\xi_{d} a^{-1 / 2}\left(\boldsymbol{\xi}^{\prime}\right)\right)\right|^{2} d \tau d \boldsymbol{\xi}^{\prime} d \xi_{d} \\
& =\int\left(a+a y^{2}\right)^{(s-2)}\left[\left(a+a y^{2}\right)^{2}+b\right] \\
& \quad \cdot \frac{\left|\widehat{u}\left(\tau, \boldsymbol{\xi}^{\prime}\right)\right|^{2}}{a^{(k+1 / 2)}\left(\boldsymbol{\xi}^{\prime}\right)}\left|\widehat{\phi}_{k}(y)\right|^{2} d \tau d \boldsymbol{\xi}^{\prime} d y \\
& \leq c \int a^{s-k-5 / 2}\left(a^{2}+b\right)\left(1+y^{2}\right)^{s-2}\left[1+y^{4} a^{2} /\left(a^{2}+b\right)\right] \\
& \quad \cdot\left|\widehat{u}\left(\tau, \boldsymbol{\xi}^{\prime}\right)\right|^{2}\left|\widehat{\phi}_{k}(y)\right|^{2} d y d \tau d \boldsymbol{\xi}^{\prime} \\
& \leq c_{1} \int\left(a^{s-k-1 / 2}+b a^{s-k-5 / 2}\right)\left|\widehat{u}\left(\tau, \boldsymbol{\xi}^{\prime}\right)\right|^{2} d \tau d \boldsymbol{\xi}^{\prime} .
\end{aligned}
$$

These equalities imply (3.30). The equalities $\gamma_{k} \circ \beta_{k}=I$ and $\gamma_{j} \circ \beta_{k}=0, j \neq k$, follow from the definitions (3.1) and (3.31) for $\gamma_{k}$ and $\beta_{k}$, respectively.

Remark 3.1. Note that for $1 / 2<s-k<5 / 2$, we have

$$
\mathcal{H}^{(s-k-1 / 2)}\left(\mathbb{R}^{d}\right) \stackrel{\subsetneq}{\ddagger} L^{2}\left(\mathbb{R} ; H^{s-k-1 / 2}\left(\mathbb{R}^{d-1}\right)\right) \cap H^{\frac{2 s-2 k-1}{4}}\left(\mathbb{R} ; L^{2}\left(\mathbb{R}^{d-1}\right)\right)
$$


and

$$
\begin{aligned}
& \mathcal{H}^{(s-k-1 / 2)}\left(\mathbb{R}^{d}\right) \stackrel{\subsetneq}{\neq} L^{2}\left(\mathbb{R} ; H^{s-k-1 / 2}\left(\mathbb{R}^{d-1}\right)\right) \\
& \cap H^{\frac{2 s-2 k-1}{2 s}}\left(\mathbb{R} ; H^{(1-2 / s)(s-k-1 / 2)}\left(\mathbb{R}^{d-1}\right)\right)
\end{aligned}
$$

(in other words, the space of restrictions obtained in Lemma 3.1 is wider than the domain of the extension operator in Lemma 3.4). Indeed, the proof of the first inclusion amounts to that of the inequality

$$
a^{s-k-1 / 2}+b^{(2 s-2 k-1) / 4} \leq C\left[a^{s-k-1 / 2}+b a^{s-k-5 / 2}\right],
$$

which, upon absorbing the term $a^{s-k-1 / 2}$ on the left side by that on the right side, can be rewritten as

$$
\left[b a^{s-k-5 / 2}\right]^{(2 s-2 k-1) / 4} a^{(5 / 2-s+k)(2 s-2 k-1) / 4} \leq C\left[a^{s-k-1 / 2}+b a^{s-k-5 / 2}\right] .
$$

Since $(2 s-2 k-1) / 4<1$, the last inequality is valid by virtue of Young's inequality. Similarly, the proof of the second inclusion amounts to that of the inequality

$$
a^{s-k-1 / 2}+b^{(s-k-1 / 2) / s} a^{(1-2 / s)(s-k-1 / 2)} \leq C\left[a^{s-k-1 / 2}+b a^{s-k-5 / 2}\right] .
$$

Upon absorbing the term $a^{s-k-1 / 2}$ on the left side by that on the right side and then dividing the inequality by $a^{s-k-5 / 2}$, we see that the last inequality is equivalent to

$$
b^{(s-k-1 / 2) / s} a^{(2 k+1) / s} \leq C\left[a^{2}+b\right],
$$

which again is valid by virtue of Young's inequality.

Let $a\left(\boldsymbol{\xi}^{\prime}\right)$ and $b(\tau)$ be defined as in (3.6) and let $\widehat{u}\left(\tau, \boldsymbol{\xi}^{\prime}\right)$ denote the Fourier transform of $u\left(t, \mathbf{x}^{\prime}\right)$. We introduce the following function spaces:

$$
B^{s}\left(\mathbb{R}^{d}\right)=\left\{u\left(t, \mathbf{x}^{\prime}\right),\left(t, \mathbf{x}^{\prime}\right) \in \mathbb{R} \times \mathbb{R}^{d-1}:\|u\|_{B^{s}\left(\mathbb{R}^{d}\right)}<\infty\right\},
$$

where

$$
\|u\|_{B^{s}\left(\mathbb{R}^{d}\right)}^{2}=\int_{\mathbb{R}^{d}}\left(a^{2}\left(\boldsymbol{\xi}^{\prime}\right)+b(\tau)\right)^{s / 2}\left|\widehat{u}\left(\tau, \boldsymbol{\xi}^{\prime}\right)\right|^{2} d \tau d \boldsymbol{\xi}^{\prime}
$$

and

$$
B^{s, k}\left(\mathbb{R}^{d}\right)=\left\{u\left(t, \mathbf{x}^{\prime}\right) \in L^{2}\left(\mathbb{R} \times \mathbb{R}^{d-1}\right):\|u\|_{B^{s, k}\left(\mathbb{R}^{d}\right)}<\infty\right\},
$$

where

$$
\|u\|_{B^{s, k}\left(\mathbb{R}^{d}\right)}=\int_{\mathbb{R}^{d}}\left(a^{2}\left(\boldsymbol{\xi}^{\prime}\right)+b^{\frac{2}{s}}(\tau) a^{\frac{2(s-2)}{s}}\left(\boldsymbol{\xi}^{\prime}\right)\right)^{\frac{2 s-2 k-1}{4}}\left|\widehat{u}\left(\tau, \boldsymbol{\xi}^{\prime}\right)\right|^{2} d \tau d \boldsymbol{\xi}^{\prime} .
$$

Lemma 3.5. Let $k \geq 0$ be an integer and $s \in \mathbb{R}$. Assume $s-k \leq 5 / 2$. Then, there exists a continuous operator

$$
\beta_{k}: B^{s-k-1 / 2}\left(\mathbb{R}^{d}\right) \rightarrow \mathcal{H}^{(s)}\left(\mathbb{R}^{d+1}\right) \quad \text { if } \quad s \geq 2
$$

and

$$
\beta_{k}: B^{s, k}\left(\mathbb{R}^{d}\right) \rightarrow \mathcal{H}^{(s)}\left(\mathbb{R}^{d+1}\right) \quad \text { if } \quad 1 / 2<s<2
$$

such that $\gamma_{k} \circ \beta_{k} u=u$ and $\gamma_{j} \circ \beta_{k} u=0, j \neq k$, where $\gamma_{k}$ is the operator (3.1). 
Proof. Let $s \geq 2$. We define the operator $\beta_{k}$ by formulae similar to (3.31)-(3.32):

$$
\widetilde{\beta_{k} u}\left(\tau, \boldsymbol{\xi}^{\prime}, x_{d}\right)=\left(a^{2}+b\right)^{-k / 4} \phi_{k}\left(\left(a^{2}+b\right)^{1 / 4} x_{d}\right) \widehat{u}\left(\tau, \boldsymbol{\xi}^{\prime}\right)
$$

and

$$
\widehat{\beta_{k} u}(\tau, \boldsymbol{\xi})=\left(a^{2}+b\right)^{-(k+1) / 4} \widehat{u}\left(\tau, \boldsymbol{\xi}^{\prime}\right) \widehat{\phi}_{k}\left(\left(a^{2}+b\right)^{-1 / 4} \xi_{d}\right) .
$$

By the definition (2.4) of the norm of $\mathcal{H}^{(s)}\left(\mathbb{R}^{d+1}\right)$ and by (3.36),

$$
\left\|\beta_{k} u\right\|_{\mathcal{H}^{(s)}\left(\mathbb{R}^{d+1}\right)}^{2}=\int_{\mathbb{R}^{d}} A\left(\tau, \boldsymbol{\xi}^{\prime}\right)\left(a^{2}+b\right)^{-(k+1) / 2}\left|\widehat{u}\left(\tau, \boldsymbol{\xi}^{\prime}\right)\right|^{2} d \tau d \boldsymbol{\xi}^{\prime},
$$

where

$$
A\left(\tau, \boldsymbol{\xi}^{\prime}\right)=\int_{\mathbb{R}}\left[\left(a+\xi_{d}^{2}\right)^{s}+b\left(a+\xi_{d}^{2}\right)^{s-2}\right]\left|\widehat{\phi}_{k}\left(\left(a^{2}+b\right)^{-1 / 4} \xi_{d}\right)\right|^{2} d \xi_{d} .
$$

Making the change of variable $y=\left(a^{2}+b\right)^{-1 / 4} \xi_{d}$ in (3.37) and taking into account the fact that, for an arbitrary $N>0$,

$$
\left|\widehat{\phi}_{k}(y)\right|^{2} \leq C_{N}(1+y)^{-N},
$$

we obtain

$$
\begin{aligned}
& A\left(\tau, \boldsymbol{\xi}^{\prime}\right) \leq C\left(a^{2}+b\right)^{1 / 4} \int_{\mathbb{R}} \frac{\left(a+\left(a^{2}+b\right)^{1 / 2} y^{2}\right)^{s-2}\left(a^{2}+b+\left(a^{2}+b\right) y^{4}\right)}{\left(1+y^{2}\right)^{N}} d y \\
& \quad \leq C\left(a^{2}+b\right)^{(2 s+1) / 4} \int_{0}^{\infty}\left[y^{2}+a /\left(a^{2}+b\right)^{1 / 2}\right]^{s-2}\left(1+y^{4}\right) /\left(1+y^{2}\right)^{N} d y \\
& \quad \leq C\left(a^{2}+b\right)^{(2 s+1) / 4} .
\end{aligned}
$$

This inequality proves the continuity of the operator (3.33).

Consider now the case $1 / 2<s<2$. We define the operator $\beta_{k}$ as follows:

$$
\widetilde{\beta_{k} u}\left(\tau, \boldsymbol{\xi}^{\prime}, x_{d}\right)=M^{-k / 4} \phi_{k}\left(\Lambda x_{d}\right) \widehat{u}\left(\tau, \boldsymbol{\xi}^{\prime}\right)
$$

and

$$
\widehat{\beta_{k} u}(\tau, \boldsymbol{\xi})=M^{-k / 4} \Lambda^{-1} \widehat{\phi_{k}}\left(\Lambda^{-1} \xi_{d}\right) \widehat{u}\left(\tau, \boldsymbol{\xi}^{\prime}\right),
$$

where

$$
\begin{aligned}
& M=a^{2}\left(\boldsymbol{\xi}^{\prime}\right)+b^{2 / s}(\tau) a^{2(s-2) / s}\left(\boldsymbol{\xi}^{\prime}\right), \\
& \Lambda=a^{(s-2) /(2 s)}\left(\boldsymbol{\xi}^{\prime}\right)\left[a^{2}\left(\boldsymbol{\xi}^{\prime}\right)+b(\tau)\right]^{1 /(2 s)} .
\end{aligned}
$$

Then we obtain, as above,

$$
\left\|\beta_{k} u\right\|_{\mathcal{H}^{(s)}\left(\mathbb{R}^{d+1}\right)}^{2}=\int_{\mathbb{R}^{d+1}} A\left(\tau, \boldsymbol{\xi}^{\prime}\right) M^{-k / 2}\left|\widehat{u}\left(\tau, \boldsymbol{\xi}^{\prime}\right)\right|^{2} d \tau d \boldsymbol{\xi}^{\prime}
$$

with

$$
\begin{aligned}
& A\left(\tau, \boldsymbol{\xi}^{\prime}\right)=\int_{\mathbb{R}}\left(\left(a+\xi_{d}^{2}\right)^{s}+b\left(a+\xi_{d}^{2}\right)^{s-2}\right) \Lambda^{-2}\left|\widehat{\phi}_{k}\left(\Lambda^{-1} \xi_{d}\right)\right|^{2} d \xi_{d} \\
& =\int_{\mathbb{R}}\left(\left(a+\Lambda^{2} y^{2}\right)^{s}+b\left(a+\Lambda^{2} y^{2}\right)^{s-2}\right) \Lambda^{-1}\left|\widehat{\phi}_{k}(y)\right|^{2} d y \\
& =\int_{\mathbb{R}}\left[\Lambda^{2 s-1}\left(\frac{a}{\Lambda^{2}}+y^{2}\right)^{s}+b \Lambda^{2 s-5}\left(\frac{a}{\Lambda^{2}}+y^{2}\right)\right]\left|\widehat{\phi}_{k}(y)\right|^{2} d y \\
& \sim \Lambda^{2 s-1}+b \Lambda^{2 s-5} .
\end{aligned}
$$


Substituting (3.39) into the last relation, we obtain

$$
\begin{aligned}
& A\left(\tau, \boldsymbol{\xi}^{\prime}\right) \sim a^{\frac{(2 s-1)(s-2)}{2 s}}\left(a^{2}+b\right)^{\frac{2 s-1}{2 s}}+b a^{\frac{(2 s-5)(s-2)}{2 s}}\left(a^{2}+b\right)^{\frac{2 s-5}{2 s}} \\
& \sim a^{s-\frac{1}{2}}+b^{1-\frac{1}{2 s}} a^{(s-2)\left(1-\frac{1}{2 s}\right)}+b\left(a^{s}+b a^{s-2}\right)^{\frac{2 s-5}{2 s}} .
\end{aligned}
$$

This last result obviously will lead us to the desired relation

$$
A\left(\tau, \boldsymbol{\xi}^{\prime}\right) \sim a^{s-\frac{1}{2}}+b^{1-\frac{1}{2 s}} a^{(s-2)\left(1-\frac{1}{2 s}\right)} \sim\left(a^{2}+b^{\frac{2}{s}} a^{(s-2) \frac{2}{s}}\right)^{\frac{2 s-1}{4}}
$$

if we can show that

$$
b\left(a^{s}+b a^{s-2}\right)^{\frac{2 s-5}{2 s}} \leq C\left(a^{s-\frac{1}{2}}+b^{1-\frac{1}{2 s}} a^{(s-2)\left(1-\frac{1}{2 s}\right)}\right) .
$$

The estimate (3.42) is equivalent to the inequality

$$
\begin{aligned}
b & \leq C\left(a^{s-\frac{1}{2}}+b^{1-\frac{1}{2 s}} a^{(s-2)\left(1-\frac{1}{2 s}\right)}\right)\left(a^{\frac{5}{2}-s}+b^{\frac{5-2 s}{2 s}} a^{\frac{(s-2)(5-2 s)}{2 s}}\right) \\
& =C\left(a^{2}+b^{2 / s} a^{2(s-2) / s}+\text { two other nonnegative terms }\right) .
\end{aligned}
$$

Using Young's inequality, we have $b a^{2(2-s) / s} \leq C\left(a^{4 / s}+b^{2 / s}\right)$, which, upon dividing by $a^{2(2-s) / s}$ on both sides, implies (3.43), or equivalently, (3.42).

From relations (3.40), (3.41), and (3.39), we deduce (3.34).

Remark 3.2. For $1 / 2<s-k<5 / 2$ the spaces of restrictions in (3.3)-(3.4) coincide with the spaces in (3.33)-(3.34) on which the extension operators $\beta_{k}$ are defined. Note that the spaces in (3.33)-(3.34) and the extension operators $\beta_{k}$ are also well defined for $s-k \leq 1 / 2$.

With the help of standard partition of unity techniques, we obtain from Lemmas 3.4 and 3.5 extension results for functions defined on $\Sigma=\mathbb{R} \times \partial \Omega$ (extension into functions defined on $Q=\mathbb{R} \times \Omega$ ).

The spaces $B^{s}(\Sigma)$ and $B^{s, k}(\Sigma)$ are defined from $B^{s}\left(\mathbb{R}^{d}\right)$ and $B^{s, k}\left(\mathbb{R}^{d}\right)$, respectively, with the help of standard partition of unity techniques.

Theorem 3.2. Let $k \geq 0$ be an integer and $s \in \mathbb{R}$. Then, there exist continuous extension operators

$$
\beta_{k}: \mathcal{H}^{(s-k-1 / 2)}(\Sigma) \rightarrow \mathcal{H}^{(s)}(Q)
$$

and

$$
\beta_{k}: B^{s-k-1 / 2}(\Sigma) \rightarrow \mathcal{H}^{(s)}(Q), \quad \text { if } \quad s \geq 2
$$

and

$$
\beta_{k}: B^{s, k}(\Sigma) \rightarrow \mathcal{H}^{(s)}(Q), \quad \text { if } 3 / 2<s<2,
$$

such that $\gamma_{k} \circ \beta_{k} v=v, \gamma_{j} \circ \beta_{k} v=0, j \neq k$, where $\gamma_{k}$ is the restriction operator which maps a function $u(t, \mathbf{x})$ defined for $(t, \mathbf{x}) \in \mathbb{R} \times \Omega$ to the function $\gamma_{k} u \equiv$ $\left.\frac{\partial^{k} u(t, \mathbf{x})}{\partial n^{k}}\right|_{\mathbf{x} \in \Sigma \equiv \mathbb{R} \times \partial \Omega}$; here $\frac{\partial^{k}}{\partial n^{k}}$ is the derivative of order $k$ with respect to the unit outward normal on $\partial \Omega$.

Theorem 3.2 can be proved from Lemmas 3.4 and 3.5 by standard partition of unity techniques. 


\section{RESTRICTION RESUlts FOR SOLENOIDAL VECTOR FIELDS}

4.1. Some results concerning a curl elliptic problem. Our aim is to obtain restriction results for vector fields $\mathbf{u} \in \mathcal{V}^{(s)}\left(Q_{T}\right)$ (see the definition in (2.5)) on the lateral surface $\Sigma_{T}$ of the cylinder $Q_{T}$. Roughly speaking, we do the following: we make the decomposition $\mathbf{u}=\mathbf{u}_{\tau}+u_{n} \mathbf{n}$ in a neighborhood of $\Sigma_{T}$, where $\mathbf{n}$ is the unit outward-pointing normal vector field to $\Sigma_{T}$ and $\mathbf{u}_{\tau}$ is the tangential component of u. We recall that

$$
\operatorname{curl} \mathbf{u}=\left(\frac{\partial u_{2}}{\partial x_{3}}-\frac{\partial u_{3}}{\partial x_{2}}, \frac{\partial u_{2}}{\partial x_{3}}-\frac{\partial u_{3}}{\partial x_{2}}, \frac{\partial u_{2}}{\partial x_{3}}-\frac{\partial u_{3}}{\partial x_{2}}\right) .
$$

To obtain restriction results for $\mathbf{u}_{\tau}$, we simply apply the results of Section 3 above. To obtain precise restriction results for $u_{n}$, we first apply to $\mathbf{u}$ the operator $\mathbf{c u r l}^{-1}$ (which has to be defined) and obtain the vector field curl ${ }^{-1} \mathbf{u} \in \mathcal{H}^{(s+1)}\left(Q_{T}\right)$. Then, using results from Section 3, we can characterize the restriction $\gamma_{\tau}\left(\mathbf{c u r l}^{-1} \mathbf{u}\right)$, where $\gamma_{\tau}$ is the composition of the restriction operator and the tangential projection. Then, we apply the operator $d_{\tau}$ which in local coordinates $\left(t, x_{1}, x_{2}\right)$ on $\Sigma_{T}$ can be written as $d_{\tau} \mathbf{v}=\partial v_{2} / \partial x_{1}-\partial v_{1} / \partial x_{2}$, where $\mathbf{v}=\left(v_{1}\left(t, x_{1}, x_{2}\right), v_{2}\left(t, x_{1}, x_{2}\right)\right)$ is a tangential vector field to $\Sigma_{T}$. One can see that, for smooth $\mathbf{u}, d_{\tau} \gamma_{\tau} \mathbf{c u r l}^{-1} \mathbf{u}=\gamma_{n} \mathbf{u}$, where $\gamma_{n}$ is the composition of the restriction operator and the projection onto the vector field of outer normals to $\Sigma_{T}$. This construction gives the precise restriction results.

The operator $(\mathbf{c u r l})^{-1}$ is not defined on the whole space

$$
\mathbf{V}^{s}(\Omega)=\left\{\mathbf{u} \in\left[H^{s}(\Omega)\right]^{3}: \operatorname{div} \mathbf{u}=0\right\}
$$

First, we study the restriction problem for a certain subspace of $\mathbf{V}^{s}(\Omega)$; the general case will be considered later in Subsection 4.4.

It is well known (see, for example, [12]) that for any $\mathbf{u} \in \mathbf{V}^{s}(\Omega), s \geq 0$, the trace $\gamma_{n} \mathbf{u}$ (see the definition of $\gamma_{n}$ in $(2.9)$ ) is well defined and $\gamma_{n} \mathbf{u} \in H^{s-1 / 2}(\partial \Omega)$. We set for $s \geq 0$

$$
\widehat{\mathbf{V}}^{s}(\Omega)=\left\{\mathbf{u} \in \mathbf{V}^{s}(\Omega): \int_{\Gamma_{j}} \gamma_{n} \mathbf{u} d \mathbf{x}^{\prime}=0, \quad j=1, \cdots, J\right\} .
$$

Let us consider the boundary value problem

$$
\begin{array}{cl}
\operatorname{curl} \mathbf{v}=\mathbf{u}, & \mathbf{x} \in \Omega, \\
\operatorname{div} \mathbf{v}=0, & \mathbf{x} \in \Omega,
\end{array}
$$

and

$$
\gamma_{n} \mathbf{v}=0, \quad \mathbf{x} \in \partial \Omega .
$$

We consider (4.2) as spaces for the right-hand side of problem (4.3)-(4.5). We need to introduce the space for solutions of problem (4.3)-(4.5). Define

$$
H_{0}^{s}(\Omega)=\text { closure of } C_{0}^{\infty} \text { under the } H^{s}(\Omega) \text { norm, } s>0,
$$

and

$$
\mathbf{V}_{0}^{0}(\Omega)=\left\{\mathbf{v} \in \mathbf{V}^{0}(\Omega): \gamma_{n} \mathbf{v}=\mathbf{0}\right\}
$$

We have the following well-known Weyl decomposition (see, e.g., 8] and [12]):

$$
\mathbf{L}^{2}(\Omega)=\mathbf{V}_{0}^{0}(\Omega) \oplus \nabla H^{1}(\Omega)
$$


where $\nabla H^{1}(\Omega)=\left\{\nabla p: p \in H^{1}(\Omega)\right\}$. Evidently, $\nabla H^{1}(\Omega) \subset$ ker curl . Note that, generally speaking, $\mathbf{V}_{0}^{0}(\Omega) \cap$ ker curl $\neq\{\mathbf{0}\}$. Indeed, the following orthogonal decomposition in $\mathbf{L}^{2}(\Omega)$ holds (see [3] and [12] Appendix 1, pp. 458-471]):

$$
\mathbf{V}_{0}^{0}(\Omega)=\mathbf{W}^{0}(\Omega) \oplus \mathbf{H}_{c},
$$

where $\mathbf{H}_{c}=\mathbf{V}_{0}^{0} \cap$ ker curl is a finite-dimensional subspace of $\mathbf{H}^{1}(\Omega)$. $\mathbf{H}_{c}$ consists of vector fields $\nabla p(\mathbf{x})$, where $p(\mathbf{x})$ are multi-valued functions satisfying $\Delta p=0$ and $\left.(\partial p / \partial n)\right|_{\partial \Omega}=0$; for details see [12, Appendix 1]). We have the following relations for the div and curl operators.

Lemma 4.1. The following relations hold:

a)

$$
\operatorname{curl} \mathbf{H}^{1}(\Omega)=\widehat{\mathbf{V}}^{0}(\Omega)
$$

and

b)

$$
\mathbf{H}^{m}(\Omega)=\left\{\mathbf{u} \in \mathbf{L}^{2}(\Omega): \operatorname{curl} \mathbf{u} \in \mathbf{H}^{m-1}(\Omega),\right.
$$

$$
\left.\operatorname{div} \mathbf{u} \in \mathbf{H}^{m-1}(\Omega), \gamma_{n} \mathbf{u} \in \mathbf{H}^{m-1 / 2}(\partial \Omega)\right\} \quad \forall m \geq 1
$$

( $m$ is an integer).

Proof. See [3] and [12, Appendix 1].

Using the space $\mathbf{W}^{0}$ defined in (4.6), we introduce the following spaces:

$$
\begin{aligned}
\mathbf{W}^{s}(\Omega)=\mathbf{W}^{0}(\Omega) & \cap \mathbf{H}^{s}(\Omega) \\
& \text { for } s \geq 0 \text { equipped with the } \mathbf{H}^{s}(\Omega) \text { norm }
\end{aligned}
$$

and the following subspaces of $\mathbf{H}^{-s}(\Omega)$ :

$$
\begin{aligned}
& \mathbf{W}^{-s}(\Omega)=\text { the closure of } \mathbf{W}^{0}(\Omega) \text { under the } \mathbf{H}^{-s}(\Omega) \text { norm, } \\
& \qquad\|\mathbf{u}\|_{-s}=\sup _{\mathbf{v} \in \mathbf{H}_{0}^{s}(\Omega)} \frac{\langle\mathbf{u}, \mathbf{v}\rangle}{\|\mathbf{v}\|_{\mathbf{H}_{0}^{s}(\Omega)}} \text { for } 0<s<1 / 2,
\end{aligned}
$$

where the duality pairing $\langle\cdot, \cdot\rangle$ is generated by the scalar product in $\mathbf{L}^{2}(\Omega)$.

Finally, for $s \in(0,3 / 2)$ we define

$$
\widehat{\mathbf{V}}^{-s}(\Omega)=\text { the closure of } \widehat{\mathbf{V}}^{0}(\Omega) \text { under the } \mathbf{H}^{-s}(\Omega) \text { norm. }
$$

Lemma 4.2. The following inclusion is true:

$$
\operatorname{curl} \mathbf{H}_{0}^{1}(\Omega) \equiv\left\{\mathbf{u}: \mathbf{u}=\operatorname{curl} \mathbf{v}, \mathbf{v} \in \mathbf{H}_{0}^{1}(\Omega)\right\} \subset \mathbf{V}_{0}^{0}(\Omega) .
$$

Lemma 4.3. Assume that $\mathbf{u} \in \mathbf{W}^{1}(\Omega)$ and $\mathbf{c u r l} \mathbf{u} \in \mathbf{W}^{0}(\Omega)$. Then there exists a $\widetilde{p} \in H^{2}(\Omega)$ such that $\mathbf{u}-\nabla \widetilde{p} \in \mathbf{H}_{0}^{1}(\Omega)$.

These lemmas will be proved later, in Subsection 4.2.

We now prove the existence and uniqueness of solutions for problem (4.3)-(4.5).

Theorem 4.1. Let $s>-3 / 2$ and $s \neq-1 / 2$. Then, the operator

$$
\text { curl : } \mathbf{W}^{s+1}(\Omega) \rightarrow \widehat{\mathbf{V}}^{s}(\Omega)
$$

is an isomorphism. Moreover, the operator

$$
\operatorname{curl}: \widehat{\mathbf{V}}^{s+1}(\Omega) \rightarrow \widehat{\mathbf{V}}^{s}(\Omega)
$$

is surjective. 
Proof. Assertion a) of Lemma 4.1 and the definition (4.6)-(4.7) of the spaces $\mathbf{W}^{s}$ imply that the operator curl : $\mathbf{W}^{1}(\Omega) \rightarrow \widehat{\mathbf{V}}^{0}(\Omega)$ is surjective and ker curl $\cap$ $\mathbf{W}^{1}(\Omega)=\{\mathbf{0}\}$. Hence, by the Banach theorem, the inverse operator curl ${ }^{-1}$ : $\widehat{\mathbf{V}}^{0}(\Omega) \rightarrow \mathbf{W}^{1}(\Omega)$ is defined and is continuous. Therefore, the operator curl : $\mathbf{W}^{1}(\Omega) \rightarrow \widehat{\mathbf{V}}^{0}(\Omega)$ is an isomorphism. Assertion b) of Lemma 4.1 implies that if a right-hand side $\mathbf{u} \in \widehat{\mathbf{V}}^{0}(\Omega)$ for problem (4.3)-(4.5) belongs to $\mathbf{H}^{m-1}(\Omega)$, then the solution $\mathbf{v} \in \mathbf{W}^{1}(\Omega)$ belongs to $\mathbf{H}^{m}(\Omega)$. Thus, the restriction to $\mathbf{W}^{m}(\Omega)$ of the operator curl : $\mathbf{W}^{1}(\Omega) \rightarrow \widehat{\mathbf{V}}^{0}(\Omega)$ yields the operator curl $: \mathbf{W}^{m}(\Omega) \rightarrow \widehat{\mathbf{V}}^{m-1}(\Omega)$, which is an isomorphism. Applying the interpolation theorems from [9] to the operators curl : $\mathbf{W}^{1}(\Omega) \rightarrow \widehat{\mathbf{V}}^{0}(\Omega)$ and curl : $\mathbf{W}^{m}(\Omega) \rightarrow \widehat{\mathbf{V}}^{m-1}(\Omega)$, we see that the operator (4.8) is an isomorphism for every $s \geq 0$.

Next, we prove that $\mathbf{H}_{0}^{1}(\Omega) \cap \mathbf{H}_{c}=\{\mathbf{0}\}$, where $\mathbf{H}_{c}$ is the space defined in (4.6). If $\nabla q \in \mathbf{H}_{0}^{1}(\Omega) \cap \mathbf{H}_{c}$, then $\Delta q=0$ and $\left.(\nabla q)\right|_{\partial \Omega}=0$. By the theorem on the uniqueness of solutions of the Cauchy problem for the Laplacian operator (i.e., the problem $\Delta q=0$ in $\Omega,\left.q\right|_{\Gamma_{j}}=$ const. $j$ for $j=1, \cdots, J$, and $\left.\frac{\partial q}{\partial n}\right|_{\partial \Omega}=0$ ), we see that $q \equiv$ constant for $\mathbf{x} \in \Omega$. Hence $\nabla q=\mathbf{0}$. Note that, for any $\mathbf{v} \in \mathbf{H}_{0}^{1}(\Omega)$ and $\nabla q \in \mathbf{H}_{c}$ (recalling that $\mathbf{H}_{c} \subset \mathbf{H}^{1}(\Omega)$ ),

$$
\int_{\Omega} \operatorname{curl} \mathbf{v} \cdot \nabla q d \mathbf{x}=\int_{\Omega} \mathbf{v} \cdot \operatorname{curl} \nabla q d \mathbf{x}=0 .
$$

Therefore, taking into account (4.6) and Lemma 4.2, we deduce that $\operatorname{curl} \mathbf{H}_{0}^{1}(\Omega) \subset$ $\mathbf{W}^{0}(\Omega)$. By virtue of $(4.8)$ with $s=0$ we have that for each $\mathbf{v} \in \mathbf{W}^{0}(\Omega) \subset \widehat{\mathbf{V}}^{0}(\Omega)$ there exists a $\mathbf{u} \in \mathbf{W}^{1}(\Omega)$ such that $\operatorname{curl} \mathbf{u}=\mathbf{v}$. By Lemma 4.3 there exists a $p \in H^{2}(\Omega)$ such that $\mathbf{u}-\nabla p \in \mathbf{H}_{0}^{1}(\Omega)$. Since $\operatorname{curl}(\mathbf{u}-\nabla p)=\mathbf{v}$, we have proved the equality $\operatorname{curl} \mathbf{H}_{0}^{1}(\Omega)=\mathbf{W}^{0}(\Omega)$.

Consider the operator

$$
\operatorname{curl}: \mathbf{H}_{0}^{1}(\Omega) \rightarrow \mathbf{L}^{2}(\Omega)
$$

and its adjoint operator

$$
\operatorname{curl}^{*}: \mathbf{L}^{2}(\Omega) \rightarrow \mathbf{H}^{-1}(\Omega) .
$$

Note that, using integration by parts, we see that

$$
\int_{\Omega}(\operatorname{curl} \mathbf{v}) \cdot \mathbf{w} d \mathbf{x}=\int_{\Omega} \mathbf{v} \cdot(\operatorname{curl} \mathbf{w}) d \mathbf{x} \quad \forall \mathbf{v}, \mathbf{w} \in \mathbf{H}_{0}^{1}(\Omega),
$$

i.e., the operator curl is formally self-adjoint. Thus we define curl as an operator from $\mathbf{L}^{2}(\Omega)$ to $\mathbf{H}^{-1}(\Omega)$ by $\mathbf{c u r l} \equiv \mathbf{c u r l}^{*}$, where $\mathbf{c u r l}^{*}$ is the operator (4.11). Let us prove that

$$
\operatorname{curl}^{*} \mathbf{L}^{2}(\Omega)=\operatorname{curl} \mathbf{L}^{2}(\Omega)=\widehat{\mathbf{V}}^{-1}(\Omega) .
$$

For each $\mathbf{u} \in \mathbf{L}^{2}(\Omega)$ there exists a sequence $\left\{\mathbf{u}_{k}\right\} \subset \mathbf{H}_{0}^{1}(\Omega)$ which converges to $\mathbf{u}$ in $\mathbf{L}^{2}(\Omega)$ as $k \rightarrow \infty$. Then curl $\mathbf{u}_{k} \rightarrow \operatorname{curl} \mathbf{u}$ in $\mathbf{H}^{-1}(\Omega)$. Since $\left\{\operatorname{curl} \mathbf{u}_{k}\right\} \subset \mathbf{W}^{0}(\Omega) \subset$ $\widehat{\mathbf{V}}^{0}(\Omega)$, we deduce from the definition of $\widehat{\mathbf{V}}^{-1}(\Omega)$ that curl $\mathbf{u} \in \widehat{\mathbf{V}}^{-1}(\Omega)$. Thus

$$
\operatorname{curl} \mathbf{L}^{2}(\Omega) \subset \widehat{\mathbf{V}}^{-1}(\Omega) .
$$

Since $\mathbf{H}_{0}^{1}(\Omega) \cap \mathbf{H}_{c}(\Omega)=\{\mathbf{0}\}$ (see above), ker curl in the space $\mathbf{H}_{0}^{1}(\Omega)$ equals $\{\nabla p$ : $\left.p \in L^{2}(\Omega), \nabla p \in \mathbf{H}_{0}^{1}(\Omega)\right\}$. If $\left.\nabla p\right|_{\partial \Omega}=\mathbf{0}$, then on each component $\Gamma_{i}$ of $\partial \Omega$ the 
equality $\left.p\right|_{\Gamma_{i}}=C_{i}$ holds, where each $C_{i}$ is a constant. Thus,

$$
\operatorname{ker}_{\mathbf{H}_{0}^{1}(\Omega)} \mathbf{c u r l}=\left\{\nabla p: p \in H^{2}(\Omega),\left.\partial_{n} p\right|_{\partial \Omega}=\mathbf{0},\left.p\right|_{\Gamma_{i}}=C_{i}\right\} .
$$

Let $\nabla p \in \operatorname{ker}_{\mathbf{H}_{0}^{1}(\Omega)}$ curl and $\mathbf{u} \in \widehat{\mathbf{V}}^{-1}(\Omega)$. Note that $\widehat{\mathbf{V}}^{1}(\Omega)$ is dense in $\widehat{\mathbf{V}}^{0}(\Omega)$. To see this, for a given $\mathbf{u} \in \widehat{\mathbf{V}}^{0}(\Omega)$, we first use Lemma 4.1a) to solve problem (4.3)-(4.5) to obtain a solution $\mathbf{v} \in \mathbf{H}^{1}(\Omega)$. Then, we choose a sequence $\left\{\mathbf{w}_{n}\right\} \subset$ $\mathbf{H}^{2}(\Omega)$ that converges to $\mathbf{v}$ in $\mathbf{H}^{1}(\Omega)$. Clearly, $\left\{\operatorname{curl} \mathbf{w}_{n}\right\} \subset \widehat{\mathbf{V}}^{1}(\Omega)$ and $\left\{\operatorname{curl}_{n}\right\}$ converges to $\mathbf{u}$ in $\mathbf{L}^{2}(\Omega)$. By the definition of $\widehat{\mathbf{V}}^{-1}(\Omega)$ and the density of $\widehat{\mathbf{V}}^{1}(\Omega)$ in $\widehat{\mathbf{V}}^{0}(\Omega)$, there exists a sequence $\left\{\mathbf{u}_{k}\right\} \subset \widehat{\mathbf{V}}^{1}(\Omega) \subset \widehat{\mathbf{V}}^{0}(\Omega)$ such that $\mathbf{u}_{k} \rightarrow \mathbf{u}$ in $\mathbf{H}^{-1}(\Omega)$ as $k \rightarrow \infty$. Integration by parts yields

$$
\begin{aligned}
& \langle\mathbf{u}, \nabla p\rangle=\lim _{k \rightarrow \infty} \int_{\Omega} \mathbf{u}_{k} \cdot \nabla p d \mathbf{x} \\
& =-\lim _{k \rightarrow \infty} \int_{\Omega} p \operatorname{div} \mathbf{u}_{k} d \mathbf{x}+\sum_{i} \lim _{k \rightarrow \infty} \int_{\Gamma_{j}} C_{j} \mathbf{u}_{k} \cdot \mathbf{n} d s=0 .
\end{aligned}
$$

Hence, $\widehat{\mathbf{V}}^{-1}(\Omega) \perp \operatorname{ker}_{\mathbf{H}_{0}^{1}(\Omega)}$ curl. This relation, together with the well-known fact $\operatorname{curl} \mathbf{L}^{2}(\Omega)=\left[\operatorname{ker}_{\mathbf{H}_{0}^{1}(\Omega)} \mathbf{c u r l}\right]^{\perp}$ and (4.13), implies (4.12).

Equalities (4.12) and (4.6) and Weyl's decomposition yield the assertion of Theorem 4.1 for the case $s=-1$. We obtain the same assertion for $s \in(-1,-1 / 2) \cup$ $(-1 / 2,0)$ with the help of the interpolation theorem.

For $s \in(1,3 / 2)$, the operator curl : $\mathbf{H}_{0}^{s}(\Omega) \rightarrow \mathbf{H}^{s-1}(\Omega)$ has the adjoint curl $^{*}=$ curl : $\mathbf{H}^{1-s}(\Omega) \rightarrow \mathbf{H}^{-s}(\Omega)$ thanks to the well-known fact $\mathbf{H}^{s-1}(\Omega)=\mathbf{H}_{0}^{s-1}(\Omega)$ for $s \in(1,3 / 2)$. Since $\mathbf{H}_{0}^{s}(\Omega)=\mathbf{H}_{0}^{1}(\Omega) \cap \mathbf{H}^{s}(\Omega)$ for $1<s<3 / 2$,

$$
\operatorname{curl} \mathbf{H}_{0}^{s}(\Omega)=\operatorname{curl}\left[\mathbf{H}_{0}^{1}(\Omega) \cap \mathbf{H}^{s}(\Omega)\right]=\mathbf{W}^{s-1}(\Omega) \equiv \mathbf{W}^{0}(\Omega) \cap \mathbf{H}^{s-1}(\Omega),
$$

and therefore, $\operatorname{curl} \mathbf{H}_{0}^{s}(\Omega)$ is closed in $\mathbf{H}^{s-1}(\Omega)$. Hence by a well-known theorem, $\operatorname{curl}^{1-s}(\Omega)=\left[\operatorname{ker}_{\mathbf{H}_{0}^{s}(\Omega)} \mathbf{c u r l}\right]^{\perp}$. Then, analogously to the proof for the case of $s=1$, we complete the proof.

To prove an analog of Theorem 4.1 in the case of $s=-1 / 2$ we need to introduce new function spaces involving $H_{00}^{1 / 2}(\Omega)$. Let $\rho(\mathbf{x}) \in C^{\infty}(\bar{\Omega})$ be such that $\rho(\mathbf{x})>0$ for all $\mathbf{x} \in \Omega$ and $\rho(\mathbf{x})=\operatorname{dist}(\mathbf{x}, \partial \Omega)$ for $\mathbf{x}$ sufficiently close to $\partial \Omega$, where $\operatorname{dist}(\mathbf{x}, \partial \Omega)$ is the Euclidean distance from $\mathbf{x}$ to $\partial \Omega$. We recall from [9] that

$$
H_{00}^{1 / 2}(\Omega)=\left\{u \in H^{1 / 2}(\Omega): \rho^{-1 / 2} u \in L^{2}(\Omega)\right\}
$$

with the norm defined by

$$
\|u\|_{H_{00}^{1 / 2}(\Omega)}^{2}=\|u\|_{H^{1 / 2}(\Omega)}^{2}+\left\|\rho^{-1 / 2} u\right\|_{L^{2}(\Omega)}^{2},
$$

and $H_{00}^{-1 / 2}(\Omega)$ is the dual space of $H_{00}^{1 / 2}(\Omega)$, i.e., it is the completion of $L^{2}(\Omega)$ under the norm

$$
\|u\|_{H_{00}^{-1 / 2}(\Omega)}=\inf _{0 \neq \phi \in H_{00}^{1 / 2}(\Omega)} \frac{\langle u, \phi\rangle}{\|\phi\|_{H_{00}^{1 / 2}(\Omega)}} .
$$

We denote by $\mathbf{H}_{00}^{1 / 2}(\Omega)$ and $\mathbf{H}_{00}^{-1 / 2}(\Omega)$ the vector counterparts of $H_{00}^{1 / 2}(\Omega)$ and $H_{00}^{-1 / 2}(\Omega)$, respectively. Now, we define

$$
\widehat{\mathbf{V}}_{00}^{-1 / 2}(\Omega)=\text { the closure of } \widehat{\mathbf{V}}^{0}(\Omega) \text { under the } \mathbf{H}_{00}^{-1 / 2}(\Omega) \text { norm. }
$$


Clearly $\widehat{\mathbf{V}}_{00}^{-1 / 2}(\Omega)$ is a subspace of $\mathbf{H}_{00}^{-1 / 2}(\Omega)$ equipped with the $\mathbf{H}_{00}^{-1 / 2}(\Omega)$ norm.

Proposition 4.1. The operator curl : $\mathbf{W}^{1 / 2}(\Omega) \rightarrow \widehat{\mathbf{V}}_{00}^{-1 / 2}(\Omega)$ is an isomorphism. Moreover, the operator curl : $\widehat{\mathbf{V}}^{1 / 2}(\Omega) \rightarrow \widehat{\mathbf{V}}_{00}^{-1 / 2}(\Omega)$ is surjective.

Proof. As is well known, $H_{00}^{-1 / 2}(\Omega)$ is the intermediate space of order $1 / 2$ between $H^{-1}(\Omega)$ and $L^{2}(\Omega)$ : i.e., $H_{00}^{-1 / 2}(\Omega)=\left[H^{-1}(\Omega), L^{2}(\Omega)\right]_{1 / 2}$. (For a proof of this fact and for the definition of intermediate spaces, see [9, Ch.1, §2.1, §12.2].) This implies that $\mathbf{V}_{00}^{-1 / 2}(\Omega)=\left[\mathbf{V}^{-1}(\Omega), \mathbf{V}^{0}(\Omega)\right]_{1 / 2}$. Thus, the desired results follow from interpolation theorems (see [9, Ch.1, §5.1]) and the assertions of Theorem 4.1 for the cases $s=0$ and $s=-1$.

We introduce the spaces

$$
\widehat{\mathcal{V}}^{(s)}\left(Q_{T}\right)=\left\{\mathbf{u} \in L^{2}\left(0, T ; \widehat{\mathbf{V}}^{s}(\Omega)\right): \partial_{t} \mathbf{u} \in L^{2}\left(0, T ; \widehat{\mathbf{V}}^{s-2}(\Omega)\right)\right\}
$$

and

$$
\widehat{\mathcal{W}}^{(s)}\left(Q_{T}\right)=\left\{\mathbf{u} \in L^{2}\left(0, T ; \mathbf{W}^{s}(\Omega)\right): \partial_{t} \mathbf{u} \in L^{2}\left(0, T ; \mathbf{W}^{s-2}(\Omega)\right\}\right.
$$

Theorem 4.2. Let $s>1 / 2$. Then, for an arbitrary $\mathbf{u} \in \widehat{\mathcal{V}}^{(s)}\left(Q_{T}\right)$, there exists a unique solution $\mathbf{v} \in \widehat{\mathcal{W}}^{(s+1)}\left(Q_{T}\right)$ for the problem $(4.3)-(4.5)$ (where $t$ is a parameter), and the following estimate holds:

$$
\|\mathbf{v}\|_{\widehat{\mathcal{W}}^{(s+1)}\left(Q_{T}\right)} \leq C\|\mathbf{u}\|_{\widehat{\mathcal{V}}^{(s)}\left(Q_{T}\right)},
$$

where $C$ does not depend on $\mathbf{u}$.

Proof. If $s-2 \neq-1 / 2$, then the desired assertion follows easily from Theorem 4.1 and the definitions $(4.14)-(4.15)$. If $s-2=-1 / 2$, then since $H_{00}^{1 / 2}(\Omega) \subset$ $H^{1 / 2}(\Omega) \subset L^{2}(\Omega) \subset H^{-1 / 2}(\Omega) \subset H_{00}^{-1 / 2}(\Omega)$, where all embeddings are continuous, we deduce that $\widehat{\mathbf{V}}^{-1 / 2}(\Omega) \subset \widehat{\mathbf{V}}_{00}^{-1 / 2}(\Omega)$ with a continuous embedding. Using the last embedding, Proposition 4.1, and (4.14)-(4.15), we again obtain the desired assertion.

4.2. Special local coordinates. Let $\Gamma=\Gamma_{i}$ be a connected component of $\partial \Omega$; see (1.5). We consider the bounded domain

$$
\Theta \equiv \Theta_{i}=\{\mathbf{x} \in \Omega: \operatorname{dist}(\mathbf{x}, \Gamma)<\delta\} \quad\left(\Gamma=\Gamma_{i}\right),
$$

where $\delta>0$ is small enough. We introduce in $\Theta$ special local coordinates.

Lemma 4.4. Define

$$
y_{3}(\mathbf{x})=\operatorname{dist}(\mathbf{x}, \Gamma), \quad \mathbf{x} \in \Theta .
$$

There exists a finite covering $\left\{U_{j}\right\}$ of $\Theta$ such that in each $U_{j}$ there exists a local coordinate system $\left(y_{1}(\mathbf{x}), y_{2}(\mathbf{x}), y_{3}(\mathbf{x})\right), y_{3}$ being defined by $(4.17)$, which is oriented as $\left(x_{1}, x_{2}, x_{3}\right)$ and satisfies the condition

$$
\nabla y_{j}(\mathbf{x}) \cdot \nabla y_{k}(\mathbf{x})=\delta_{j k}, \quad j, k=1,2,3,
$$

where $\delta_{j k}$ is the Kronecker symbol. 
Proof. We choose $\delta$ in (4.16) so small that $\Theta_{i} \cap \Gamma_{j}=\emptyset$ for $i \neq j$, the function (4.17) is infinitely differentiable for $\mathbf{x} \in \Theta_{i}$, and for any $\mathbf{x} \in \Theta$ the distance from $\mathbf{x}$ to $\Gamma=\Gamma_{i}$ is achieved at a unique point $\mathbf{x}_{0} \in \Gamma=\Gamma_{i}$. Let $\mathbf{x}_{0} \in \Gamma$, and let $\left(\mathbf{e}_{1}, \mathbf{e}_{2}, \mathbf{e}_{3}\right)$ be the orthonormal basis with the origin at $\mathbf{x}_{0}$, which is oriented the same way as the orthogonal basis corresponding to initial global coordinates $\left(x_{1}, x_{2}, x_{3}\right)$ in $\Omega$; $\mathbf{e}_{j} \in T_{\mathbf{x}_{0}} \Gamma, j=1,2$, are vectors tangent to $\Gamma$ and $\mathbf{e}_{3}$ is orthogonal to $\Gamma$ directed towards the interior of $\Omega$. Shifting the initial origin to the point $\mathbf{x}_{0}$ and rotating the basis corresponding to the global coordinates, we can suppose that $\left(x_{1}, x_{2}, x_{3}\right)$ are the coordinates associated with the basis $\left(\mathbf{e}_{1}, \mathbf{e}_{2}, \mathbf{e}_{3}\right)$. It is sufficient to construct the local coordinates $y_{1}(\mathbf{x})$ and $y_{2}(\mathbf{x})$ in a small neighborhood of the interval $\left\{\mathbf{c e}_{3}\right.$ : $c>0\} \cap \Theta=\left\{c \mathbf{e}_{3}: 0<c<\delta\right\}$.

Let $\widehat{C}_{k}$ be the geodesic on the manifold $\Gamma$ going out from $\mathbf{x}_{0}$ in the directions $\mathbf{e}_{k}$ and $-\mathbf{e}_{k}, k=1,2$. There exists a unique $\widehat{C}_{k}, k=1,2$, which is defined in a neighborhood of $\mathbf{x}_{0}$ and satisfies this condition. Let $C_{3}(t, \mathbf{z})$ be a curve going out from a point $\mathbf{z} \in \Gamma$ which is the solution of the problem

$$
\partial_{t} C_{3}(t, \mathbf{z})=\nabla y_{3}\left(C_{3}(t, \mathbf{z})\right), \quad C_{3}(0, \mathbf{z})=\mathbf{z} .
$$

This solution is well defined for $C_{3} \in \Theta$. Moreover, the curve $t \rightarrow C_{3}(t, \mathbf{z})$ coincides with the normal to $\Gamma$ going out from $\mathbf{z} \in \Gamma$. Denote

$$
\begin{aligned}
\Xi_{k 3}=\{\mathbf{x} \in \Theta & : \text { there exist } t \in(0, \delta) \text { and } \\
\mathbf{z} & \left.\in \widehat{C}_{k} \text { such that } \mathbf{x}=C_{3}(t, \mathbf{z})\right\}, \quad k=1,2 .
\end{aligned}
$$

The surface $\Xi_{13}$ divides $\Theta$ into two parts: $\Theta_{2+}\left(\mathbf{e}_{2} \in \Theta_{2+}\right)$ and $\Theta_{2-}\left(-\mathbf{e}_{2} \in \Theta_{2-}\right)$. Analogously, $\Xi_{23}$ divides $\Theta$ into $\Theta_{1+}\left(\mathbf{e}_{1} \in \Theta_{1+}\right)$ and $\Theta_{1-}\left(-\mathbf{e}_{1} \in \Theta_{1-}\right)$. For $\mathbf{x} \in \Theta_{2+}$ close to the set $\left\{c \mathbf{e}_{3}: c>0\right\} \cap \Theta$, we define $y_{2}(\mathbf{x})$ as the geodesic distance on the manifold

$$
\Gamma_{y_{3}(\mathbf{x})}=\left\{\mathbf{z} \in \Theta: y_{3}(\mathbf{z})=y_{3}(\mathbf{x})\right\}
$$

from $\mathbf{x}$ to the surface $\Xi_{13}$. (The metric on $\Gamma_{y_{3}(\mathbf{x})}$ is generated by the metric of the enveloping Euclidean space.) In other words, among all geodesics on the manifold (4.19) starting from $\mathbf{x}$ and terminating on $\Xi_{13}$ we choose the geodesic $C_{2}(\mathbf{x})$ having the minimum length. This minimum length is $y_{2}(\mathbf{x})$, by definition. If $\mathbf{x} \in \Theta_{2-}$, then $y_{2}(\mathbf{x})$ is the same geodesic distance with the minus sign. Note that the vector $\nabla y_{2}(\mathbf{x})$ is tangent to the curve $C_{2}(\mathbf{x}) \subset \Gamma_{y_{3}(\mathbf{x})}$, and therefore

$$
\nabla y_{3}(\mathbf{x}) \perp \nabla y_{2}(\mathbf{x}) \text {. }
$$

Define the curve

$$
\widetilde{C}_{1}(\mathbf{x})=\Gamma_{y_{3}(\mathbf{x})} \cap\left\{\mathbf{z} \in \Theta: y_{2}(\mathbf{z})=y_{2}(\mathbf{x})\right\} .
$$

Let $C_{1}(\mathbf{x})$ denote the part of $\widetilde{C}_{1}(\mathbf{x})$ which goes out of $\mathbf{x}$ and ends on $\Xi_{23}$. We define $y_{1}(\mathbf{x})$ as the length of $C_{1}(\mathbf{x})$ if $\mathbf{x} \in \Theta_{1+}$ and as the negative of the length of $C_{1}(\mathbf{x})$ if $\mathbf{x} \in \Theta_{1-}$. Evidently, $\nabla y_{1}(\mathbf{x})$ is tangent to $C_{1}(\mathbf{x})$. Thus, by (4.21),

$$
\nabla y_{1}(\mathbf{x}) \perp \nabla y_{2}(\mathbf{x}) \quad \text { and } \quad \nabla y_{1}(\mathbf{x}) \perp \nabla y_{3}(\mathbf{x}) .
$$

By the definition of $y_{i}(\mathbf{x}), i=1,2,3$ (these were all defined by means of some distance functions), the following equalities hold:

$$
\left|\nabla y_{i}(\mathbf{x})\right|=1, \quad i=1,2,3 .
$$

These equalities, together with (4.20) and (4.22), yield (4.18). Thus, we have that $\left(y_{1}(\mathbf{x}), y_{2}(\mathbf{x}), y_{3}(\mathbf{x})\right)$ is the desired local coordinate system defined in a neighborhood 
$U_{\mathbf{x}_{0}}$ of the curve $C_{3}\left(t, \mathbf{x}_{0}\right)$. Using the closedness of the set $\Gamma=\Gamma_{i}$, we may choose a finite covering satisfying the desired properties.

Let us calculate the metric tensor $g_{i j}(\mathbf{y})$ in the local coordinates $\mathbf{y}(\mathbf{x})$ constructed in Lemma 4.4. Relation (4.18) implies that the map $\mathbf{y}(\mathbf{x})=\left(y_{1}(\mathbf{x}), y_{2}(\mathbf{x}), y_{3}(\mathbf{x})\right)$ has the inverse $\mathbf{x}(\mathbf{y})$ and $\frac{\partial x_{k}}{\partial y_{l}}=\frac{\partial y_{l}}{\partial x_{k}}$. Since $g_{k l}(\mathbf{y})$ can be found from the relation

$$
d s^{2}=\sum_{i=1}^{3} d x_{i}^{2}=\frac{\partial x_{i}}{\partial y_{k}} \frac{\partial x_{i}}{\partial y_{l}} d y_{k} d y_{l}
$$

we have

$$
g_{k l}(\mathbf{y})=\frac{\partial x_{i}}{\partial y_{k}} \frac{\partial x_{i}}{\partial y_{l}}=\nabla y_{k}(\mathbf{x}) \cdot \nabla y_{l}(\mathbf{x})=\delta_{k l} .
$$

Proof of Lemma 4.2. As is well known (see, e.g., [11. Ch. VI, §4]), the operator curl : $\mathbf{H}_{0}^{1}(\Omega) \rightarrow \mathbf{L}^{2}(\Omega)$ depends on the Euclidean structure and orientation. Hence, in the local coordinates $\left(y_{1}(\mathbf{x}), y_{2}(\mathbf{x}), y_{3}(\mathbf{x})\right)$ constructed in Lemma 4.4, the operator curl on any $U_{i}$ has the usual form:

$$
\operatorname{curl} \mathbf{w}(\mathbf{y})=\left(\frac{\partial w_{2}}{\partial y_{3}}-\frac{\partial w_{3}}{\partial y_{2}}, \frac{\partial w_{3}}{\partial y_{1}}-\frac{\partial w_{1}}{\partial y_{3}}, \frac{\partial w_{1}}{\partial y_{2}}-\frac{\partial w_{2}}{\partial y_{1}}\right)
$$

where $\mathbf{w}(\mathbf{y})$ is the expression in local coordinates $\mathbf{y}(\mathbf{x})$ of a vector field $\mathbf{u}(\mathbf{x}) \in$ $\mathbf{H}_{0}^{1}(\Omega)$. Evidently, $\mathbf{w}(\mathbf{y}) \in\left[H^{1}\left(\Omega \cap U_{i}\right)\right]^{3}$,

$$
\left.\left.\mathbf{w}(\mathbf{y})\right|_{\Gamma} \equiv \mathbf{w}(\mathbf{y})\right|_{y_{3}=0}=\mathbf{0}
$$

and $\left(y_{1}, y_{2}, 0\right)$ are the local coordinates on $\Gamma=\Gamma_{i}$. Hence, (4.25) yields

$$
\frac{\partial w_{1}}{\partial y_{2}}-\left.\frac{\partial w_{2}}{\partial y_{1}}\right|_{y_{3}=0}=0
$$

which implies that

$$
\left.(\operatorname{curl} \mathbf{w}, \mathbf{n})\right|_{\Gamma}=0
$$

where we recall that $\mathbf{n}=\mathbf{e}_{3}$ is the orthogonal vector field to $\Gamma$. Since div curl $\mathbf{u}=0$, (4.26) implies that curl $\mathbf{u} \in \mathbf{V}_{0}^{0}(\Omega)$.

Proof of Lemma 4.3. As above, we consider $\mathbf{u}(\mathbf{x})$ in local coordinates $\left(y_{1}(\mathbf{x}), y_{2}(\mathbf{x})\right.$, $\left.y_{3}(\mathbf{x})\right)$ constructed in the proof of Lemma 4.4 on an element $U_{i}$ of a finite covering $\left\{U_{i}\right\}$ for a component $\Gamma_{j}$ of $\partial \Omega$. We denote by $\mathbf{w}(\mathbf{y})$ the expression of $\mathbf{u}(\mathbf{x})$ in the local coordinates. Since $\mathbf{u} \in \mathbf{W}^{1}(\Omega)$ and curl $\mathbf{u} \in \mathbf{W}^{0}(\Omega)$, by virtue of $(4.24)$ we have

$$
\left.w_{3}\left(y_{1}, y_{2}, y_{3}\right)\right|_{y_{3}=0}=0
$$

and

$$
\left.\left(\frac{\partial w_{1}\left(y_{1}, y_{2}, y_{3}\right)}{\partial y_{2}}-\frac{\partial w_{2}\left(y_{1}, y_{2}, y_{3}\right)}{\partial y_{1}}\right)\right|_{y_{3}=0}=0
$$

Set

$$
p_{0}\left(y_{1}, y_{2}\right)=\int_{\left(y_{1}^{0} . y_{2}^{0}\right)}^{\left(y_{1}, y_{2}\right)} w_{1}\left(z_{1}, z_{2}, 0\right) d z_{1}+w_{2}\left(z_{1}, z_{2}, 0\right) d z_{2},
$$

where $\left(y_{1}^{0} \cdot y_{2}^{0}\right)$ is a fixed point on $\Gamma_{j}$ and the integration is taken over a curve connecting the points $\left(y_{1}^{0} \cdot y_{2}^{0}\right)$ and $\left(y_{1} . y_{2}\right)$ - thanks to (4.28) the integral is independent 
of the choice of the connecting curve. Evidently, the definition of $p_{0}$ can be extended onto all of $\Gamma_{j}$. Since $\mathbf{w} \in \mathbf{W}^{1}(\Omega) \subset \mathbf{H}^{1}(\Omega)$, we have $p_{0} \in H^{3 / 2}\left(\Gamma_{j}\right)$. Let $p\left(y_{1}, y_{2}, y_{3}\right)$ be a function defined on the set $\Theta_{j}$ as defined in (4.16) that satisfies

$$
p \in H^{2}(\Theta),\left.\quad p\right|_{\Gamma}=p_{0} \quad \text { and }\left.\quad \frac{\partial p}{\partial y_{3}}\right|_{\Gamma}=0 .
$$

We choose a $\varphi\left(y_{3}\right) \in C^{\infty}(0, \delta)$ satisfying $\varphi\left(y_{3}\right)=1$ for $0<y_{3}<\delta / 3$ and $\varphi\left(y_{3}\right)=0$ for $2 \delta / 3<y_{3}<\delta$. We define $p_{j}(\mathbf{y})=\varphi\left(y_{3}\right) p(\mathbf{y})$ (with $j$ being the index of $\Gamma_{j}$ ). We extend $p_{j}$ from $\Theta_{j}$ into $\Omega$ with $p_{j}(\mathbf{x})=0$ for $\mathbf{x} \in \Omega \backslash \Theta_{j}$. Set $\widehat{P}(\mathbf{x})=\sum_{j} p_{j}(\mathbf{x})$. Evidently, $\mathbf{v}(\mathbf{x})=\mathbf{u}(\mathbf{x})-\nabla \widehat{p}(\mathbf{x}) \in \mathbf{H}_{0}^{1}(\Omega)$.

4.3. Restriction theorems. Recall that $\gamma, \gamma_{\tau}, \gamma_{n}$ are the restriction operators defined in (2.9) and above.

Theorem 4.3. Let $\Omega \subset \mathbb{R}^{3}$. Then, the operator

$$
\begin{aligned}
\gamma=\left(\gamma_{\tau}, \gamma_{n}\right): \widehat{\mathcal{V}}^{(s)}\left(Q_{T}\right) & \rightarrow G_{\tau}^{s}\left(\Sigma_{T}\right) \times G_{n}^{s}\left(\Sigma_{T}\right) \\
& \text { for } s>1 / 2, s \neq 3 / 2, s \neq 5 / 2
\end{aligned}
$$

is continuous, where $G_{\tau}^{s}\left(\Sigma_{T}\right), G_{n}^{s}\left(\Sigma_{T}\right)$, and $\widehat{\mathcal{V}}^{s}\left(Q_{T}\right)$ are the spaces defined in (2.10)(2.11) and (4.14).

Proof. The trace operator $\gamma \mathbf{u}=\left(\gamma_{\tau} \mathbf{u}, \gamma_{n} \mathbf{u}\right)$ in the local coordinates introduced in the proof of Lemma 4.4 can be rewritten as follows:

$$
\gamma \mathbf{u}=\left(\left.w_{1}\right|_{y_{3}=0},\left.w_{2}\right|_{y_{3}=0},\left.w_{3}\right|_{y_{3}=0}\right), \quad \text { i.e., } \gamma_{\tau} \mathbf{u}=\left.\left(w_{1}, w_{2}\right)\right|_{y_{3}=0} .
$$

To estimate the component $\gamma_{\tau} \mathbf{u}$ of the trace operator we note that

$$
w_{i} \in \mathcal{H}^{(s)}\left((0, T) \times \Theta_{i}\right), \quad i=1,2 .
$$

We can extend $w_{i}$ up to functions belonging to $\mathcal{H}^{(s)}\left(\mathbb{R}^{d+1}\right)$. Then, we apply Lemmas 3.1 and 3.3 to deduce the continuity of the $\gamma_{\tau}$ component of the operator (4.29).

To estimate the $\gamma_{n}$ component of (4.29), we return to the original global coordinates. We define by $\operatorname{curl}^{-1} \mathbf{u}=\mathbf{v}$ the solution $\mathbf{v} \in \widehat{\mathcal{W}}^{(s+1)}\left(Q_{T}\right) \subset\left[\mathcal{H}^{(s+1)}\left(Q_{T}\right)\right]^{3}$ of the problem (4.3)-(4.5), with $\mathbf{u}$ being the right-hand side. The tangential component of $\mathbf{v}$ on $\Sigma_{T}$ in the local coordinates introduced in the proof of Lemma 4.4 can be written as $\gamma_{\tau} \mathbf{v}=\left(v_{1}\left(y_{1}, y_{2}, 0\right), v_{2}\left(y_{1}, y_{2}, 0\right)\right)$. Applying the operator $d_{\tau}$, we have $\left.d_{\tau} \mathbf{v}=\partial_{2} v_{1}\left(y_{1}, y_{2}, 0\right)-\partial_{1} v_{2}\left(y_{1}, y_{2}, 0\right)\right)$. Simple transformations yield

$$
\begin{aligned}
\gamma_{n} \mathbf{u}=u_{3}\left(y_{1}, y_{2}, 0\right) & =\gamma_{n} \mathbf{c u r l}^{\circ} \circ \mathbf{c u r l}^{-1} \mathbf{u} \\
& =\partial_{2} v_{1}\left(y_{1}, y_{2}, 0\right)-\partial_{1} v_{2}\left(y_{1}, y_{2}, 0\right) .
\end{aligned}
$$

Let us consider the case $1 \leq s<3 / 2$ (other cases can be treated similarly). Applying to (4.30) Definition (2.11), assertion (3.27), and Theorem 4.2, we obtain

$$
\begin{aligned}
& \left\|\gamma_{n} \mathbf{u}\right\|_{G_{n}^{s}\left(\Sigma_{T}\right)}=\left\|\left.\left(\partial_{2} v_{1}-\partial_{1} v_{2}\right)\right|_{\Sigma_{T}}\right\|_{L^{2}\left(0, T ; H^{s-1 / 2}(\partial \Omega)\right) \cap H^{\frac{2 s+1}{4}}\left(0, T ; H^{-1}(\partial \Omega)\right)} \\
& \leq C\left\|\left.\mathbf{v}\right|_{\Sigma_{T}}\right\|_{L^{2}\left(0, T ; H^{s+1 / 2}(\partial \Omega)\right) \cap H^{\frac{2 s+1}{4}}\left(0, T ; L^{2}(\partial \Omega)\right)} \\
& \leq C\|\mathbf{v}\|_{H^{(s+1)}\left(Q_{T}\right)}=C\|\mathbf{v}\|_{\widehat{\mathcal{W}}^{(s+1)}\left(Q_{T}\right)} \leq C\|\mathbf{u}\|_{\widehat{\mathcal{V}}^{(s)}\left(Q_{T}\right)} .
\end{aligned}
$$


Theorem 4.4. Let $\Omega \subset \mathbb{R}^{3}$. Then, the following restriction operators are bounded:

$$
\gamma=\left(\gamma_{\tau}, \gamma_{n}\right): \widehat{\mathcal{V}}^{(5 / 2)}\left(Q_{T}\right) \rightarrow\left[\mathcal{H}_{\ln }^{(2)}\left(\Sigma_{T}\right)\right]^{2} \times \mathcal{H}^{(2)}\left(\Sigma_{T}\right)
$$

and

$$
\begin{aligned}
& \gamma=\left(\gamma_{\tau}, \gamma_{n}\right): \widehat{\mathcal{V}}^{(3 / 2)}\left(Q_{T}\right) \\
& \quad \rightarrow\left[L^{2}\left(0, T ; H^{1}(\partial \Omega)\right) \cap H^{2 / 3}\left(0, T ; H^{-1 / 3}(\partial \Omega)\right)\right]^{2} \\
& \times\left[L^{2}\left(0, T ; H^{1}(\partial \Omega)\right) \cap H_{\ln }^{1}\left(0, T ; H^{-1}(\partial \Omega)\right) \cap H^{2 / 3}\left(0, T ; H^{-1 / 3}(\partial \Omega)\right)\right],
\end{aligned}
$$

where the logarithmic spaces in (4.31)-(4.32) were defined in (2.7)-(2.8).

Proof. We begin from (4.31). Since $\widehat{\mathcal{V}}^{(5 / 2)}\left(Q_{T}\right) \subset\left[\mathcal{H}^{(5 / 2)}\left(Q_{T}\right)\right]^{3}$, by Theorem 3.1 (see $(3.29)$ ) we have

$$
\gamma=\left(\gamma_{\tau}, \gamma_{n}\right): \widehat{\mathcal{V}}^{(5 / 2)}\left(Q_{T}\right) \subset\left[\mathcal{H}^{(5 / 2)}\left(Q_{T}\right)\right]^{3} \rightarrow\left[\mathcal{H}_{\ln }^{(2)}\left(\Sigma_{T}\right)\right]^{3} .
$$

On the other hand, as in Theorem 4.3, we reduce the study of $\gamma_{n}$ to the investigation of the operator

$$
\left.\left(w_{1}, w_{2}\right) \mapsto\left(\frac{\partial w_{1}}{\partial y_{2}}-\frac{\partial w_{2}}{\partial y_{1}}\right)\right|_{y_{3}=0} \equiv\left(\left.\frac{\partial}{\partial y_{2}} w_{1}\right|_{y_{3}=0}-\left.\frac{\partial}{\partial y_{1}} w_{2}\right|_{y_{3}=0}\right)
$$

defined on $\left[\mathcal{H}^{(7 / 2)}\left(Q_{T}\right)\right]^{2}$. By Theorem 3.1 (see (3.26)) this operator acts continuously from $\left[\mathcal{H}^{(7 / 2)}\left(Q_{T}\right)\right]^{2}$ to $\mathcal{H}^{(2)}\left(\Sigma_{T}\right)$. This last result and (4.33) yield (4.31).

To prove (4.32), we note that by (3.28) the operator

$$
\begin{aligned}
\gamma=\left(\gamma_{\tau}, \gamma_{n}\right): & \widehat{\mathcal{V}}^{(3 / 2)}\left(Q_{T}\right) \subset\left[\mathcal{H}^{(3 / 2)}\left(Q_{T}\right)\right]^{3} \\
& \rightarrow\left[L^{2}\left(0, T ; H^{1}(\partial \Omega)\right) \cap H^{2 / 3}\left(0, T ; H^{-1 / 3}(\partial \Omega)\right)\right]^{3}
\end{aligned}
$$

is bounded. Moreover, as above, the operator $\gamma_{n}$ can be represented as the following composition: $\gamma_{n}=d_{\tau} \circ \gamma \circ \mathbf{c u r l}^{-1}$. That is why, by Theorem 4.2 and (3.29), the operator

$$
\begin{aligned}
\gamma_{n}: & \widehat{\mathcal{V}}^{(3 / 2)}\left(Q_{T}\right) \stackrel{\text { curl }^{-1}}{\longrightarrow} \widehat{\mathcal{W}}^{(5 / 2)}\left(Q_{T}\right) \subset\left[\mathcal{H}^{(5 / 2)}\left(Q_{T}\right)\right]^{3} \\
& \stackrel{\gamma_{\tau}}{\longrightarrow} \mathcal{H}_{\ln }^{(2)}\left(\Sigma_{T}\right) \stackrel{d_{\tau}}{\longrightarrow}\left[L^{2}\left(0, T ; H^{1}(\partial \Omega)\right)\right]_{\ln } \cap H_{\ln }^{1}\left(0, T ; H^{-1}(\partial \Omega)\right)
\end{aligned}
$$

is continuous. This and (4.34) imply (4.32).

\subsection{The proof of Theorem 2.1.}

Proof of Theorem 2.1. Let $\mathbf{v} \in \mathcal{V}^{(s)}\left(Q_{T}\right), s>1 / 2$. Denote

$$
\int_{\Gamma_{j}} \mathbf{v} \cdot \mathbf{n} d s=q_{j}(t), \quad j=1, \cdots, J
$$

where $\Gamma_{i}$ is a connected component of $\partial \Omega($ see (1.5)). Since $\operatorname{div} \mathbf{v}=0$,

$$
\sum_{i=1}^{J} q_{i}(t)=0 .
$$

At each $t \in[0, T]$, we consider the Neumann problem

$$
-\Delta p(t, \mathbf{x})=0,\left.\quad \frac{\partial p}{\partial n}\right|_{\Gamma_{j}}=q_{j}(t), \quad j=1, \cdots, J .
$$

By virtue of (4.35) the problem (4.36) has a solution $p(t, \mathbf{x})$, and $\nabla p(t, \mathbf{x})$ is defined unambiguously. By the regularity theorem for solutions of problem (4.36), $\nabla p \in$ 
$H^{1}\left(0, T ;\left[C^{\infty}(\bar{\Omega})\right]^{3}\right) \subset \mathcal{H}^{(s)}(Q)$. Since $\operatorname{div} \nabla p=\Delta p=0$, we have that $\nabla p \in$ $\mathcal{V}^{(s)}(Q)$, so that

$$
\mathbf{v}-\nabla p \in \widehat{\mathcal{V}}^{(s)}(Q)
$$

To obtain the restriction result for $\mathbf{v}-\nabla p$, we can apply Theorems 4.3 and 4.4. Thus, we only need to obtain estimates for $\left.\nabla p\right|_{\Gamma_{i}}=q_{i}(t)$. Since $q_{i}(t)$ is constant with respect to $\mathbf{x}^{\prime} \in \Gamma_{i}$, we have that for any $s_{1} \neq s_{2}$,

$$
\left\|q_{i}(t, \cdot)\right\|_{H^{s_{1}}\left(\Gamma_{i}\right)} \leq C_{1}\left\|q_{i}(t, \cdot)\right\|_{H^{s_{2}}\left(\Gamma_{i}\right)} \leq\left\|q_{i}(t, \cdot)\right\|_{H^{s_{1}}\left(\Gamma_{i}\right)}
$$

where $C_{1}=\|1\|_{H^{s_{1}\left(\Gamma_{i}\right)}} /\|1\|_{H^{s_{2}\left(\Gamma_{i}\right)}}$ does not depend on $q_{i}$. Therefore, Theorems 4.3 and 4.4 and relations (4.31)-(4.32) yield the assertion of Theorem 2.1.

\section{EXTENSION RESULTS FOR SOLENOIDAL VECTOR FIELDS}

The plan for the proof of the extension theorem will be as follows. In the first step, for a given trace $\mathbf{u} \in \widehat{G}^{s}\left(\Sigma_{T}\right)$ (see $(2.16)-(2.17)$ ), we solve the system of equations

$$
\left.(\operatorname{curl} \mathbf{w})\right|_{\partial \Omega}=\mathbf{u}
$$

and as a result we obtain the trace data $\left.\mathbf{w}\right|_{\partial \Omega}$ for the vector field $\mathbf{w}$. Then, in the second step, using the extension results from Section 3, we construct $\mathbf{w}$ and define the desired extension $\mathbf{v}$ for $\mathbf{u}$ satisfying $\mathbf{v}=\mathbf{c u r l} \mathbf{w}$.

5.1. On a certain system of equations defined on $\partial \Omega$. To simplify notations, we assume in this subsection that the manifold $\partial \Omega$ is connected. In the case when $\partial \Omega$ is not connected, all arguments of this subsection should be applied to each connected component $\Gamma_{i}$ of $\partial \Omega=\bigcup \Gamma_{i}$. Below, we will use, on the manifold $\partial \Omega$, the local coordinates $\left(y_{1}, y_{2}\right)$ which are the restriction to $y_{3}=0$ of the local coordinates $\left(y_{1}, y_{2}, y_{3}\right)$ constructed in Lemma 4.4. By virtue of $(4.22), \nabla y_{1}(\mathbf{x}) \perp \nabla y_{2}(\mathbf{x})$ for $\mathbf{x}=\left(x_{1}, x_{2}, x_{3}\right) \in \partial \Omega \cap U_{i}$. Thus, by (4.23), the metric tensor on $\partial \Omega$ generated by the Euclidean metric of the enveloping space $\mathbb{R}^{3}$ has the form

$$
g_{k l}(\mathbf{y})=\delta_{k l}, \quad k, l=1,2,
$$

where $\delta_{k l}$ is the Kronecker symbol. In the bounded subdomain $\Theta$ of the domain $\Omega$ as defined in (4.16), we will use the local coordinates $\left(y_{1}, y_{2}, y_{3}\right)$ constructed in Lemma 4.4 .

Let

$$
\mathbf{u}=\sum_{i=1}^{3} u_{i} \frac{\partial}{\partial y_{i}}=\left(\mathbf{u}_{\tau}, u_{n}\right) \in G_{\tau}^{s}\left(\Sigma_{T}\right) \times G_{n}^{s}\left(\Sigma_{T}\right)=G^{s}\left(\Sigma_{T}\right)
$$

be a given vector field defined on $\partial \Omega$, where $\mathbf{u}_{\tau}=u_{1} \frac{\partial}{\partial y_{1}}+u_{2} \frac{\partial}{\partial y_{2}}$ and $u_{n}=u_{3} \frac{\partial}{\partial y_{3}}$. By virtue of (4.23), applying to (5.2) the operation of lowering indices (see [2, p. 170]) and then applying the operation $*$ (see [2, p. 175]), we can express the vector field (5.2) in the exterior differential form:

$$
\check{\mathbf{u}}=u_{3} d y_{1} \wedge d y_{2}-u_{2} d y_{1} \wedge d y_{3}+u_{1} d y_{2} \wedge d y_{3} .
$$

By the operation of lowering indices we express $\mathbf{w}$ defined in (5.1) as a differential form $\check{\mathbf{w}}$ on $\Theta$ which in local coordinates $\left(y_{1}, y_{2}, y_{3}\right)$ takes the form

$$
\check{\mathbf{w}}=w_{1}\left(y_{1}, y_{2}, y_{3}\right) d y_{1}+w_{2}\left(y_{1}, y_{2}, y_{3}\right) d y_{2}+w_{3}\left(y_{1}, y_{2}, y_{3}\right) d y_{3} \text {. }
$$


We also introduce a differential form $\hat{\mathbf{w}}$ on $\partial \Omega$ depending on $y_{3}$ as a parameter which in local coordinates $\left(y_{1}, y_{2}\right)$ takes the form

$$
\hat{\mathbf{w}}=w_{1}\left(y_{1}, y_{2}, y_{3}\right) d y_{1}+w_{2}\left(y_{1}, y_{2}, y_{3}\right) d y_{2} .
$$

Of course, the forms $\check{\mathbf{w}}$ and $\hat{\mathbf{w}}$ are related. We rewrite equation (5.1) for vector fields as the equation $d \check{\mathbf{w}}=\check{\mathbf{u}}$ for differential forms, which in local coordinates $\left(y_{1}, y_{2}, y_{3}\right)$ is written as follows:

$$
\begin{array}{ll}
\left(\partial_{y_{1}} w_{2}-\partial_{y_{2}} w_{1}\right) d y_{1} \wedge d y_{2}=u_{3} d y_{1} \wedge d y_{2} & \text { for } y_{3}=0 \\
\left(\partial_{y_{2}} w_{3}-\partial_{y_{3}} w_{2}\right) d y_{2} \wedge d y_{3}=u_{1} d y_{2} \wedge d y_{3} & \text { for } y_{3}=0
\end{array}
$$

and

$$
\left(\partial_{y_{1}} w_{3}-\partial_{y_{3}} w_{1}\right) d y_{1} \wedge d y_{3}=-u_{2} d y_{1} \wedge d y_{3} \quad \text { for } y_{3}=0 .
$$

Our first goal is to find the restrictions $\left.w_{i}\right|_{y_{3}=0}, i=1,2,3$, from (5.5)-(5.7). To this end, we set

$$
\left.w_{3}\right|_{y_{3}=0}=0
$$

Equations (5.6)-(5.8) imply

$$
-\partial_{y_{3}} w_{2}=u_{1} \quad \text { and } \quad \partial_{y_{3}} w_{1}=u_{2} \quad \text { for } y_{3}=0 .
$$

Thus, to find the traces for $w_{1}$ and $w_{2}$ at $y_{3}=0$, we have to solve (5.5) defined on the manifold $\partial \Omega$. This equation can be rewritten in the following invariant form:

$$
d \omega=\widetilde{u},
$$

where $\widetilde{u} \in \Lambda^{2}(\partial \Omega)$ is the given differential form, which in local coordinates has the expression

$$
\widetilde{u}=u_{3} d y_{1} \wedge d y_{2}
$$

and $\omega \in \Lambda^{1}(\partial \Omega)$ is an unknown differential form of the first order, which in local coordinates can be written as follows:

$$
\omega=\omega_{1} d y_{1}+\omega_{2} d y_{2}=\left.\hat{\mathbf{w}}\right|_{y_{3}=0} .
$$

The operator $d: \Lambda^{1}(\partial \Omega) \rightarrow \Lambda^{2}(\partial \Omega)$ in (5.10) is the usual operator of taking differentials (see [1] and [10). Equation (5.10) has a large kernel. For this reason, we supplement (5.10) with the following equation:

$$
d * \omega=0,
$$

where $*: \Lambda^{1}(\partial \Omega) \rightarrow \Lambda^{1}(\partial \Omega)$ is the conjugation operator, which in local coordinates can be written as follows (see [1] and 10]):

$$
* \omega=-\omega_{2} d y_{1}+\omega_{1} d y_{2} \quad \text { for } \omega=\omega_{1} d y_{1}+\omega_{2} d y_{2} .
$$

In local coordinates, (5.12) takes the form

$$
\left(\partial_{y_{1}} \omega_{1}+\partial_{y_{2}} \omega_{2}\right) d y_{1} \wedge d y_{2}=0 .
$$

Note that (5.9) can be written in the following invariant form:

$$
\left.\left(* \partial_{y_{3}} \hat{\mathbf{w}}\right)\right|_{y_{3}=0}=\hat{\mathbf{u}}, \quad \text { where } \hat{\mathbf{u}}=u_{1} d y_{1}+u_{2} d y_{2}
$$


and $\hat{\mathbf{w}}$ is the exterior form defined in (5.4). Recall (see [1] and [10]) that the conjugate operators

$$
*: \Lambda^{2}(\partial \Omega) \rightarrow \Lambda^{0}(\partial \Omega) \quad \text { and } \quad *: \Lambda^{0}(\partial \Omega) \rightarrow \Lambda^{2}(\partial \Omega)
$$

in local coordinates are defined as follows:

$$
\begin{aligned}
& * f=f d y_{1} \wedge d y_{2} \quad \text { if } f \in \Lambda^{0}(\partial \Omega), \\
& *\left(f d y_{1} \wedge d y_{2}\right)=f \quad \text { if } f d y_{1} \wedge d y_{2} \in \Lambda^{2}(\partial \Omega) .
\end{aligned}
$$

Then, the Laplace operator

$$
\Delta: \Lambda^{i}(\partial \Omega) \rightarrow \Lambda^{i}(\partial \Omega), \quad i=0,1,2,
$$

is defined as follows (see [1] and [10]):

$$
\Delta f=-* d * d f-d * d * f \quad \text { for } f \in \Lambda^{i}(\partial \Omega), \quad i=0,1,2 .
$$

By $L^{2}\left(\Lambda^{i}\right), i=0,1,2$, we denote the set of $\omega \in \Lambda^{i}(\partial \Omega)$ with the finite norm

$$
\|\omega\|_{L^{2}\left(\Lambda^{i}\right)}^{2}=\int_{\partial \Omega} \omega \wedge * \omega .
$$

(Note that the scalar product in $L^{2}\left(\Lambda^{i}\right)$ is defined by $\left(\omega_{1}, \omega_{2}\right)=\int_{\partial \Omega} \omega_{1} \wedge * \omega_{2}$.) In $L^{2}\left(\Lambda^{1}\right)$ we introduce the following subspaces (see [10]):

$$
\begin{gathered}
E=E(\partial \Omega)=\text { the closure of }\left\{d f: f \in C^{1}(\partial \Omega)\right\} \text { in } L^{2}\left(\Lambda^{1}\right), \\
E^{*}=E^{*}(\partial \Omega)=\text { the closure of }\left\{* d f: f \in C^{1}(\partial \Omega)\right\} \text { in } L^{2}\left(\Lambda^{1}\right),
\end{gathered}
$$

and

$$
H=H(\partial \Omega)=\left\{\omega \in L^{2}\left(\Lambda^{1}\right): \omega=d f, \text { where } f \in \Lambda^{0}(\partial \Omega) \text { and } \Delta f=0\right\} .
$$

These spaces have the following relations (see [10, pp. 196-200]):

Lemma 5.1. a) The spaces $E, E^{*}$ and $H$ are mutually orthogonal, and $L^{2}\left(\Lambda^{1}\right)=$ $E \oplus E^{*} \oplus H$.

b) $d \omega=0$ iff $\omega \in\left(E^{*}\right)^{\perp}$, and $d * \omega=0$ iff $\omega \in E^{\perp}$, where $E^{\perp}\left(\right.$ or $\left.\left(E^{*}\right)^{\perp}\right)$ is the orthogonal complement of $E$ (or $\left.E^{*}\right)$ in $L^{2}\left(\Lambda^{1}\right)$.

c) $d \omega=0$ and $d * \omega=0$ iff $\omega \in H$.

We now solve the system formed by (5.10) and (5.12).

Lemma 5.2. a) There exists a solution $\omega \in H \oplus E^{*}$ of (5.10) and (5.12) if and only if the right side $\widetilde{u} \in L^{2}\left(\Lambda^{2}\right)$ satisfies the condition

$$
\int_{\partial \Omega} \widetilde{u}=0 .
$$

b) Any solution $\omega$ of (5.10) and (5.12) admits the representation

$$
\omega=h+* d g
$$

where $h$ is an arbitrary element of $H$ and $g \in L^{2}\left(\Lambda^{0}\right)$ is the unique solution of the equation

$$
\Delta g=-* \widetilde{u}
$$

in the class $g \in L^{2}\left(\Lambda^{0}\right)$ satisfying

$$
\int_{\partial \Omega} * g=0
$$


Proof. Let

$$
D(d)=\left\{\omega \in L^{2}\left(\Lambda^{1}\right): d \omega \in L^{2}\left(\Lambda^{2}\right)\right\}
$$

be the domain of the operator $d: L^{2}\left(\Lambda^{1}\right) \rightarrow L^{2}\left(\Lambda^{2}\right)$. The Stokes formula implies that each $\widetilde{u} \in d D(d)$ satisfies (5.15). Let $\phi \in(D(d))^{\perp} \equiv\left\{\phi \in \Lambda^{2}(\partial \Omega): \int d \omega \wedge * \phi=\right.$ $0 \forall \omega \in D(d)\}$. Then, for any $\omega=p_{1} d y_{1}+p_{2} d y_{2} \in L^{2}\left(\Lambda^{1}\right)$ with $C_{0}^{\infty}$-coefficients concentrated in a parametric circle $U_{i}$, we have

$$
\begin{aligned}
0 & =\int_{\partial \Omega} d \omega \wedge * \phi=\int_{\partial \Omega}\left(\partial_{y_{1}} p_{2}-\partial_{y_{2}} p_{1}\right) \phi d y_{1} \wedge d y_{2} \\
& =\int_{\partial \Omega}\left[\partial_{y_{1}}\left(p_{2} \phi\right)-\partial_{y_{2}}\left(p_{1} \phi\right)\right] d y_{1} \wedge d y_{2}-\int_{\partial \Omega}\left(p_{2} \partial_{y_{1}} \phi-p_{1} \partial_{y_{2}} \phi\right) d y_{1} \wedge d y_{2} \\
& =-\int_{\partial \Omega}\left(p_{2} \partial_{y_{1}} \phi-p_{1} \partial_{y_{2}} \phi\right) d y_{1} \wedge d y_{2},
\end{aligned}
$$

where in the last equality we used the following formula, which is a consequence of the Stokes theorem and the assumption that $\left.p_{k}\right|_{\partial U_{i}}=0, k=1,2$ :

$$
\int_{U_{i}}\left[\partial_{y_{1}}\left(p_{2} \phi\right)-\partial_{y_{2}}\left(p_{1} \phi\right)\right] d y_{1} \wedge d y_{2}=\int_{\partial U_{i}} p_{1} \phi d y_{1}+p_{2} \phi d y_{2}=0 .
$$

Hence, $\partial_{y_{1}} \phi=0$ and $\partial_{y_{2}} \phi=0$ in any parametric circle, and thus $* \phi=$ constant.

Let $\widetilde{u} \in d D(d)$ be given. Then, by definition there exists $\omega \in L^{2}\left(\Lambda^{1}\right)$ such that

$$
d \omega=\widetilde{u} .
$$

By virtue of Lemma 5.1 we can decompose $\omega$ as follows:

$$
\omega=h+* d g+d g_{1},
$$

where $h \in H, * d g \in E^{*}$, and $d g_{1} \in E$. Substituting (5.20) into (5.19) and taking into account that $d d g_{1}=0$ and $d h=0$ (see Lemma $5.1 \mathrm{c}$ )), we have

$$
d * d g=\widetilde{u} .
$$

By virtue of (5.14) we have that if $f \in \Lambda^{0}(\partial \Omega)$, then $\Delta f=-* d * d f$. Hence, (5.21) yields (5.17).

Multiplying the corresponding sides of (5.21) and (5.17), we see that in any parametric circle $U_{i}$,

$$
\int_{U_{i}}|\Delta g|^{2} d y_{1} \wedge d y_{2}=\int_{U_{i}}|\widetilde{u}|^{2} d y_{1} \wedge d y_{2}
$$

Using this last equation, one can deduce the closedness of the set $d D(d)$ in $L^{2}\left(\Lambda^{2}\right)$ as follows. As is well known, to establish this closedness we need the following inequality in addition to the equality above:

$$
\|g\|_{L^{2}(\partial \Omega)} \leq C_{1}\|* d g\|_{L^{2}\left(\Lambda^{1}\right)} \leq C_{2}\|\widetilde{u}\|_{L^{2}\left(\Lambda^{2}\right)} .
$$

The first of these inequalities is the well-known Poincaré inequality, which is valid because of (5.18). To prove the second inequality we multiply (5.21) by $g$ and perform integration by parts. Then, by applying the Cauchy-Schwarz and Poincaré inequalities, we obtain

$$
\|* d g\|_{L^{2}\left(\Lambda^{1}\right)}^{2}=\int_{\partial \Omega} g \widetilde{u} \leq\|\widetilde{u}\|_{L^{2}\left(\Lambda^{2}\right)}\|g\|_{L^{2}(\partial \Omega)} \leq C_{1}\|\widetilde{u}\|_{L^{2}\left(\Lambda^{2}\right)}\|* d g\|_{L^{2}\left(\Lambda^{1}\right)} .
$$

Since $d D(d)$ is closed in $L^{2}\left(\Lambda^{2}\right)$ and its orthogonal complement in $L^{2}\left(\Lambda^{2}\right)$ equals $* C$, where $* C$ is the ${ }^{*}$-image of all constant functions, we have that $[d D(d)] \oplus * C=$ 
$L^{2}\left(\Lambda^{2}\right)$. This implies the solvability of (5.19) for each $\widetilde{u}$ satisfying (5.15). The equalities $d d g_{1}=0$ and (5.20) yield that there exists a solution $\omega$ of (5.19) in the form of (5.16), where $h \in H$ and $* d g \in E^{*}$. Since $\omega=h+* d g \in H \oplus E^{*}=E^{\perp}$, we conclude from Lemma $5.1 \mathrm{~b}$ ) that $\omega$ satisfies (5.12). It was shown earlier that (5.21) implies (5.17).

Suppose that $g_{1} \in L^{2}\left(\Lambda^{0}\right)$ satisfies $\Delta g_{1}=-* \widetilde{u}$. Then, $g_{0} \equiv g-g_{1}$ satisfies the equation

$$
\Delta g_{0}=0
$$

By virtue of (5.14)

$$
\begin{aligned}
0 & =\int_{\partial \Omega} g_{0} \wedge * \Delta g_{0}=\int_{\partial \Omega} g_{0} \wedge d * d g_{0} \\
& =\int_{\partial \Omega} d\left(g_{0} * d g_{0}\right)-\int_{\partial \Omega} d g_{0} \wedge * d g_{0}=-\left\|d g_{0}\right\|_{L^{2}\left(\Lambda^{1}\right)}^{2} .
\end{aligned}
$$

Hence, $d g_{0}=0$ or $g_{0}=$ constant. But, as a result of (5.18), we see that $g_{0}=0$.

5.2. Solvability of system (5.10) and (5.12) in $H^{r}\left(0, T ; H^{s}\left(\Lambda^{1}\right)\right)$. Recall that the definition of Sobolev spaces $H^{s}(\partial \Omega)=H^{s}\left(\Lambda^{0}\right)$ of functions determined on $\partial \Omega$ was given in Section 2. The Sobolev space $H^{s}\left(\Lambda^{2}\right)$ is the set of exterior forms $u \in \Lambda^{2}(\partial \Omega)$ such that $* u \in H^{s}\left(\Lambda^{0}\right)$. The Sobolev space $H^{s}\left(\Lambda^{1}\right)$ is the set of exterior forms $u \in \Lambda^{1}(\partial \Omega)$ which in each parametric circle $U_{i}$ have the form $u=$ $p_{1}(y) d y_{1}+p_{2}(y) d y_{2}$, where the coefficients $p_{j}(y), j=1,2$, belong to the Sobolev space $H^{s}\left(U_{i}\right)$.

Lemma 5.3. Let $s \in \mathbb{R}$, and assume that $\widetilde{u} \in H^{s}\left(\Lambda^{2}\right)$ satisfies the condition (5.15), which is understood in the sense of distributions if $s<0$. Then, there exists a solution $\omega \in H^{s+1}\left(\Lambda^{1}\right)$ for (5.10) and (5.12) satisfying the estimate

$$
\|\omega\|_{H^{s+1}\left(\Lambda^{1}\right)} \leq C\|\widetilde{u}\|_{H^{s}\left(\Lambda^{2}\right)} .
$$

Proof. As is well known, for $\widetilde{u} \in H^{s}\left(\Lambda^{2}\right)$ satisfying (5.15), there exists a unique solution $g \in H^{s+2}\left(\Lambda^{0}\right)$ of (5.17), and the following estimate holds:

$$
\|g\|_{H^{s+2}\left(\Lambda^{0}\right)} \leq C\|\widetilde{u}\|_{H^{s}\left(\Lambda^{2}\right)} .
$$

Set $\omega=* d g$. Then, as in the proof of Lemma 5.2, one can establish that the exterior form $\omega \in H^{s+1}\left(\Lambda^{1}\right)$ and satisfies (5.10) and (5.12). Inequality (5.22) follows from (5.23).

Lemma 5.3 yields the following assertion.

Lemma 5.4. Let $r \geq 0$ and $s \in \mathbb{R}$. Assume that $\widetilde{u} \in H^{r}\left(0, T ; H^{s}\left(\Lambda^{2}\right)\right)$ satisfies (5.15) for almost every $t \in[0, T]$. Then, there exists an element

$$
\omega \in H^{r}\left(0, T ; H^{s+1}\left(\Lambda^{1}\right)\right)
$$

which satisfies (5.10) and (5.12) for almost every $t \in[0, T]$.

Proof. By the definition of $H^{r}\left(0, T ; H^{s}\left(\Lambda^{2}\right)\right)$, we can suppose that $\widetilde{u}$ is extended into $\widetilde{u} \in H^{r}\left(\mathbb{R} ; H^{s}\left(\Lambda^{2}\right)\right.$ ) (we still denote the extended exterior form by $\widetilde{u}$ ). Let $\widehat{u}(\tau, \cdot)$ denote the Fourier transform of $\widetilde{u}$ with respect to $t$. The assumption $\widetilde{u} \in$ $H^{r}\left(\mathbb{R} ; H^{s}\left(\Lambda^{2}\right)\right)$ implies the inequality

$$
\int_{\mathbb{R}}\left(1+|\tau|^{2}\right)^{\tau}\|\widehat{u}(\tau, \cdot)\|_{H^{s}\left(\Lambda^{2}\right)}^{2} d \tau<\infty .
$$


Then, we substitute $\widehat{u}(\tau, \cdot)$ into the right hand side of (5.10) and apply Lemma 5.3 to the system (5.10) and (5.12) to obtain the solution $\widehat{\omega}(\tau, \cdot)$ of this system. Let $\omega(t, \cdot)$ be the inverse Fourier transform of $\widehat{\omega}(\tau, \cdot)$ :

$$
\omega(t, \cdot)=\left(F_{\tau \rightarrow t}^{-1} \widehat{\omega}\right)(t, \cdot) .
$$

By virtue of $(5.22)$ and $(5.24), \omega \in H^{r}\left(\mathbb{R} ; H^{s+2}\left(\Lambda^{1}\right)\right)$, and we simply need to restrict this function to the interval $(0, T)$ to complete the proof.

5.3. Final extension result. We introduce a new function space. We set

$$
\widehat{G}^{s}\left(\Sigma_{T}\right)=G_{\tau}^{s}\left(\Sigma_{T}\right) \times \widehat{G}_{n}^{s}\left(\Sigma_{T}\right) .
$$

Here, $G_{\tau}^{s}\left(\Sigma_{T}\right)$ is the space $(2.10)$, and

$$
\begin{aligned}
\widehat{G}_{n}^{s}\left(\Sigma_{T}\right)=\{ & u_{n}(t, x) \in G_{n}^{s}\left(\Sigma_{T}\right): \\
& \left.\int_{\Gamma_{j}} u_{n}\left(t, x^{\prime}\right) d x^{\prime}=0 \text { a.e. } t \in[0, T], j=1, \ldots, J\right\},
\end{aligned}
$$

where $G_{n}^{s}\left(\Sigma_{T}\right)$ is the space $(2.11)$, and the $\Gamma_{i}$ 's are the connected components of $\partial \Omega$; see $(1.5)$.

Theorem 5.1. Let $\Omega \subset \mathbb{R}^{3}$. Then for $s>1 / 2$ there exists a continuous extension operator

$$
R: \widehat{G}^{s}\left(\Sigma_{T}\right) \rightarrow \mathcal{V}^{(s)}\left(Q_{T}\right),
$$

i.e., the operator $R$ is such that $\gamma \circ R=I$, where $I: \widehat{G}^{s}\left(\Sigma_{T}\right) \rightarrow \widehat{G}^{s}\left(\Sigma_{T}\right)$ is the identity operator.

Proof. Let $\mathbf{u}$ be the trace data (5.2). We consider the system of equations (5.5)(5.7) for the corresponding exterior differential forms. This system can be reduced to the system (5.5), (5.8), and (5.9). Finding a solution $\omega$ of (5.5) is reduced to solving the system (5.10) and (5.12) written in their invariant form. Lemma 5.4 implies that there exists a solution $\omega$ of system (5.10) and (5.12), and

$$
\omega \in\left\{\begin{array}{cc}
{\left[L^{2}\left(0, T ; H^{s+1 / 2}\left(\Lambda^{1}\right)\right)\right.} & \text { for } s \geq 3 / 2, \\
\left.\cap H^{1}\left(0, T ; H^{s-3 / 2}\left(\Lambda^{1}\right)\right)\right]^{2} & \\
{\left[L^{2}\left(0, T ; H^{s+1 / 2}\left(\Lambda^{1}\right)\right)\right.} & \text { for } 1 \leq s \leq 3 / 2, \\
\left.\cap H^{(2 s+1) / 4}\left(0, T ; L^{2}\left(\Lambda^{1}\right)\right)\right]^{2} & \\
{\left[L^{2}\left(0, T ; H^{s+1 / 2}\left(\Lambda^{1}\right)\right)\right.} & \text { for } 1 / 2<s \leq 1 . \\
\left.\quad \cap H^{\frac{2 s+1}{2 s+2}}\left(0, T ; H^{\frac{(s-1)(2 s+1)}{2(s+1)}}\left(\Lambda^{1}\right)\right)\right]^{2} &
\end{array}\right.
$$

By virtue of (5.13),

$$
\hat{\mathbf{u}} \in \begin{cases}{\left[L^{2}\left(0, T ; H^{s-1 / 2}\left(\Lambda^{1}\right)\right)\right.} & s \geq 5 / 2, \\ \left.\quad \cap H^{1}\left(0, T ; H^{s-5 / 2}\left(\Lambda^{1}\right)\right)\right]^{2}, & \\ {\left[L^{2}\left(0, T ; H^{s-1 / 2}\left(\Lambda^{1}\right)\right)\right.} & 2 \leq s \leq \frac{5}{2}, \\ \left.\cap H^{(2 s-1) / 4}\left(0, T ; L^{2}\left(\Lambda^{1}\right)\right)\right]^{2}, & \\ {\left[L^{2}\left(0, T ; H^{s-1 / 2}\left(\Lambda^{1}\right)\right)\right.} & \\ \left.\quad \cap H^{\frac{2 s-1}{2 s}}\left(0, T ; H^{\left(1-\frac{2}{s}\right)\left(s-\frac{1}{2}\right)}\left(\Lambda^{1}\right)\right)\right]^{2}, & \frac{1}{2}<s \leq 2 .\end{cases}
$$

Now we want to solve the problem of extending a differential form given on $\Sigma_{T}=$ $(0, T) \times \partial \Omega$ into a differential form defined in a neighborhood $[0, T] \times \Theta$ of $\Sigma_{T}$ (see $(4.16))$. Let $\left\{U_{i}\right\}$ be a finite covering of $\partial \Omega$ that was introduced previously, and 
suppose that $\left\{\phi_{i}\right\}$ is a partition of unity subordinate to $\left\{U_{i}\right\}$. By means of the exterior forms (5.25)-(5.26), we construct the following exterior forms:

$$
\omega^{(i)}=\phi_{i} \omega \quad \text { and } \quad \hat{\mathbf{u}}^{(i)}=\phi_{i} \hat{\mathbf{u}} .
$$

Evidently,

$$
\sum_{i} \omega^{(i)}=\omega \quad \text { and } \quad \sum_{i} \hat{\mathbf{u}}^{(i)}=\hat{\mathbf{u}} .
$$

In the local coordinates $\left(y_{1}, y_{2}\right)$ which we constructed in $U_{i}$, we consider the following extension problem:

$$
\begin{aligned}
& \left.w_{j}^{(i)}(t, \mathbf{y})\right|_{y_{3}=0}=\phi_{i} \omega_{j}\left(t, y_{1}, y_{2}\right), \quad j=1,2, \\
& \left.\partial_{y_{3}} w_{1}^{(i)}(t, \mathbf{y})\right|_{y_{3}=0}=\phi_{1} u_{2}\left(t, y_{1}, y_{2}\right), \\
& \left.\partial_{y_{3}} w_{2}^{(i)}(t, \mathbf{y})\right|_{y_{3}=0}=-\phi_{2} u_{1}\left(t, y_{1}, y_{2}\right),
\end{aligned}
$$

where $\phi_{i} \omega_{1} d y_{1}+\phi_{i} \omega_{2} d y_{2}=\omega^{(i)}=\phi_{i} \omega$ and

$$
\phi_{i} u_{1} d y_{1}+\phi_{i} u_{2} d y_{2}=\hat{\mathbf{u}}^{(i)}=\phi_{i} \hat{\mathbf{u}} .
$$

Note that the spaces (5.25) are included into the corresponding domains of the operators $\beta_{0}$ from $(3.44)$, $(3.45),(3,46)$ in which $s$ is replaced by $s+1$. Also, the domain of the operator $\beta_{1}$ from (3.44), (3.45), (3.46) with $s$ changed to $s+1$ contains the corresponding spaces from (5.26). All these assertions are proved by straightforward verifications except for the space in (5.26) when $1 / 2<s \leq 2$. In the case of $1 / 2<s \leq 2$, we must prove the inclusion

$$
L^{2}\left(0, T ; H^{s-1 / 2}(\partial \Omega)\right) \cap H^{\frac{2 s-1}{2 s}}\left(0, T ; H^{(1-2 / s)(s-1 / 2)}(\partial \Omega)\right) \subset B^{s+1,1}\left(\Sigma_{T}\right),
$$

which reduces to the proof of the following estimate for the weights defining the indicated spaces:

$$
\begin{aligned}
& \left(a^{2}\left(\boldsymbol{\xi}^{\prime}\right)+b^{\frac{2}{s+1}}(\tau) a^{\frac{2(s-1)}{s+1}}\left(\boldsymbol{\xi}^{\prime}\right)\right)^{\frac{2 s-1}{4}} \\
& \quad \leq C\left(a^{s-1 / 2}\left(\boldsymbol{\xi}^{\prime}\right)+b^{\frac{2 s-1}{2 s}}(\tau) a^{(1-2 / s)(s-1 / 2)}\left(\boldsymbol{\xi}^{\prime}\right)\right),
\end{aligned}
$$

where $1 / 2<s \leq 2$. This inequality is equivalent to the following:

$$
a^{2}+b^{\frac{2}{s+1}} a^{\frac{2(s-1)}{s+1}} \leq C\left(a^{2}+b^{\frac{2}{s}} a^{\frac{2(s-2)}{s}}\right) .
$$

But the last inequality is true thanks to an estimate by Hölder's inequality:

$$
b^{\frac{2}{s+1}} a^{\frac{2(s-1)}{s+1}}=\left(b^{\frac{2}{s+1}} a^{\frac{2(s-2)}{s+1}}\right)\left(a^{\frac{2}{s+1}}\right) \leq C\left(b^{\frac{2}{s}} a^{\frac{2(s-2)}{s}}+a^{2}\right) .
$$

By Theorem 3.2, there exist $v_{j}(t, \mathbf{y}) \in \mathcal{H}^{(s+1)}\left((0, T) \times U_{i}\right), j=1,2$, satisfying (5.27). Let $\psi_{i} \in C_{0}^{\infty}\left(U_{i}\right)$ be functions satisfying

$$
\psi_{i} \phi_{i} \equiv \phi_{i}, \quad i=1, \cdots, K,
$$

$\widehat{\chi}\left(y_{3}\right) \in C^{\infty}(0, \delta), \widehat{\chi}\left(y_{3}\right)=1$ for $\mathbf{y}$ belonging to a neighborhood of 0 , and $\widehat{\chi}\left(y_{3}\right)=0$ for y belonging to a neighborhood of $\delta$, where $\delta$ is the magnitude found in (4.16). Equalities (5.27)-(5.28) yield

$$
\left.\widehat{\chi} \psi_{i} w_{j}^{(i)}(t, \mathbf{y})\right|_{y_{3}=0}=\phi_{i} \omega_{j}\left(t, y_{1}, y_{2}\right), \quad j=1,2,
$$




$$
\begin{aligned}
& \left.\widehat{\chi} \psi_{i} \partial_{y_{3}} w_{1}^{(i)}(t, \mathbf{y})\right|_{y_{3}=0}=\phi_{i} u_{2}\left(t, y_{1}, y_{2}\right), \\
& \left.\widehat{\chi} \psi_{i} \partial_{y_{3}} w_{2}^{(i)}(t, \mathbf{y})\right|_{y_{3}=0}=-\phi_{i} u_{1}\left(t, y_{1}, y_{2}\right) .
\end{aligned}
$$

Evidently, the extension problem $\left.w_{3}(t, \mathbf{y})\right|_{y_{3}=0}=0$ has the solution

$$
w_{3}^{(i)}(\tau, \mathbf{y})=\widehat{\chi}\left(y_{3}\right) \psi_{i} 0 \equiv 0 \in \mathcal{H}^{(s+1)}\left((0, T) \times U_{i}\right) .
$$

From the left-hand sides of (5.29), (5.30), and (5.31), we construct the differential form

$$
\check{\mathbf{w}}^{(i)}=\sum_{j=1}^{2} \widehat{\chi} \psi_{i} w_{j}^{(i)}(t, \mathbf{y}) d y_{j}+w_{3}^{(i)}(t, \mathbf{y}) d y_{3}
$$

that is written in local coordinates defined in $[0, T] \times U_{i} \times(0, \delta)$. But it can be extended into the whole cylinder $Q_{T}$ by zero outside that local region. Define the differential form $\theta=\sum_{i=1}^{K} d \check{\mathbf{w}}^{(i)}$. This form is defined on $Q_{T}$ with coefficients belonging to $\mathcal{H}^{(s)}\left(Q_{T}\right)$. Moreover, previous constructions yield that $\left.\theta\right|_{\Sigma_{T}}=\check{\mathbf{u}}$, where $\check{\mathbf{u}}$ is the external form (5.3). This proves Theorem $5.1 \mathrm{a}$ ).

We are now in a position to prove the main extension result.

Proof of Theorem 2.2. Let $\mathbf{u} \in G^{s}\left(\Sigma_{T}\right)$ be given, which we decompose into $\mathbf{u}=$ $\mathbf{u}_{\tau}+u_{n} \mathbf{n}$ with $\mathbf{u}_{\tau} \in G_{\tau}^{s}\left(\Sigma_{T}\right)$ and $u_{n} \in G_{n}^{s}\left(\Sigma_{T}\right)$. On each connected component $\Gamma_{j}$ of $\partial \Omega$ (see (1.5)), we set

$$
q_{j}(t) \equiv \int_{\Gamma_{j}} u_{n}\left(t, \mathbf{x}^{\prime}\right) d \mathbf{x}^{\prime}, \quad j=1, \cdots, J .
$$

$u_{n} \in G_{n}^{s}\left(\Sigma_{T}\right)$ implies that $q_{j}(t) \in H^{\sigma}(0, T)$ for each $j$, where

$$
\sigma= \begin{cases}1, & s \geq 3 / 2 \\ (2 s+1) / 4, & 1 \leq s \leq 3 / 2 \\ (2 s+1) /(2 s+2), & 1 / 2<s \leq 1\end{cases}
$$

According to (2.1)-(2.2) the space $H^{\sigma}(0, T)$ is defined as

$$
H^{\sigma}(0, T)=H^{\sigma}(\mathbb{R}) / H_{(0, T)^{\prime}}^{\sigma}(\mathbb{R})
$$

with the norm

$$
\|q\|_{H^{\sigma}(0, T)}=\inf _{E}\|E q\|_{H^{\sigma}(\mathbb{R})} \quad \forall q \in H^{\sigma}(0, T) .
$$

Thus for each $q \in H^{\sigma}(0, T)$ there exists an extension $E$ such that

$$
\|q\|_{H^{\sigma}(0, T)} \leq\|E q\|_{H^{\sigma}(\mathbb{R})} \leq C\|q\|_{H^{\sigma}(0, T)},
$$

where $C>0$ does not depend on $q$ and

$$
\|E q\|_{H^{\sigma}(\mathbb{R})}=\int_{\mathbb{R}}\left(1+|\tau|^{2}\right)^{\sigma}|\widehat{E q}(\tau)|^{2} d \tau
$$

$(\widehat{E q}$ is the Fourier transform of $E q)$. For $q_{j}$ defined in (5.32) we denote by $E_{j} q_{j}$ the extension of $q_{j}$ satisfying (5.33). From the definition (2.11) of $G_{n}^{s}\left(\Sigma_{T}\right)$ and the inclusion $u_{n} \in G_{n}^{s}\left(\Sigma_{T}\right)$ we have $\sum_{j=1}^{J} q_{j}(t)=0$ for every $t \in(0, T)$. Without loss of generality we may suppose that $\sum_{j=1}^{J} E_{j} q_{j}(t)=0$ for every $t \in \mathbb{R}$, for otherwise we can define $E_{J} q_{J}(t)=-\sum_{q=1}^{J-1} E_{j} q_{j}(t)$, and evidently (5.33) is still true for this 
newly defined $E_{J}$ with $C$ replaced by $(J-1) C$. For each $t \in \mathbb{R}$, we consider the Neumann problem

$$
\Delta p(t, \mathbf{x})=0, \quad \mathbf{x} \in \Omega,
$$

and

$$
\left.\frac{\partial p}{\partial n}\right|_{\Gamma_{j}}=E_{j} q_{j}(t), \quad j=1, \cdots, J .
$$

Since $\sum_{j=1}^{J} E_{j} q_{j}(t) \equiv 0$ for every $t \in \mathbb{R}$, problem (5.34)-(5.35) has a solution $p(t, \mathbf{x})$ such that $\nabla p(t, \mathbf{x})$ is defined unambiguously and the following inequality holds:

$$
\|\nabla p(t, \cdot)\|_{H^{s}(\Omega)}^{2} \leq C_{s} \sum_{j=1}^{J}\left|E_{j} q_{j}(t)\right|^{2} \quad \forall s \in \mathbb{R} .
$$

Let $\widehat{E_{j} q_{j}}(\tau)$ be the Fourier transform of $E_{j} q_{j}(t)$ and $\widehat{p}(\tau, \mathbf{x})$ be the Fourier transform in $t$ of $p(t, \mathbf{x})$. Evidently, $\widehat{p}(\tau, \mathbf{x})$ and $\widehat{E_{j} q_{j}}(\tau)$ satisfy $(5.34)-(5.35)$ for every $\tau \in \mathbb{R}$. Thus, analogously to (5.36), we have

$$
\|\nabla \widehat{p}(\tau, \cdot)\|_{H^{s}(\Omega)}^{2} \leq C_{s} \sum_{j=1}^{J}\left|\widehat{E_{j} q_{j}}(\tau)\right|^{2} \quad \forall s \in \mathbb{R} .
$$

Inequalities (5.33) and (5.37) imply

$$
\|\nabla p(\tau, \cdot)\|_{H^{\sigma}\left(0, T ; H^{s}(\Omega)\right)}^{2} \leq C \sum_{j=1}^{J}\left\|q_{j}\right\|_{H^{\sigma}(0, T)}^{2} .
$$

Evidently the vector field $\nabla p(t, \mathbf{x})$ is solenoidal. Let us consider the restriction of $\nabla p(t, \mathbf{x})$ on $\Sigma$ :

$$
\left.\nabla p(t, \mathbf{x})\right|_{\Sigma}=(\nabla p)_{\tau}+(\nabla p)_{n} \mathbf{n},
$$

where $(\nabla p)_{n}=\left.\frac{\partial p}{\partial n}\right|_{\Sigma}=\left\{q_{j}\right\}$ is the normal component of $\nabla p$ on $\Sigma$ and $(\nabla p)_{\tau}=$ $\left.(\nabla p)\right|_{\Sigma}-q_{j} \mathbf{n}$ is the tangential component of $\nabla p$ on $\Sigma$. Inequalities (5.36) and (5.38) imply that for each $\gamma \in \mathbb{R}$

$$
(\nabla p)_{\tau} \in H^{1}\left(0, T ; \mathbf{H}^{\gamma}(\partial \Omega)\right) \quad \text { and } \quad(\nabla p)_{n} \in H^{1}\left(0, T: H^{\gamma}(\partial \Omega)\right) .
$$

We define the extension operator $R$ for $\left.(\nabla p)\right|_{\Sigma}$ by the formula

$$
R\left(\left.(\nabla p)\right|_{\Sigma}\right)=\nabla p(t, \mathbf{x}) .
$$

If $\mathbf{u} \in G^{s}\left(\Sigma_{T}\right)$, then by (5.39) we have $\left.(\mathbf{u}-\nabla p)\right|_{\Sigma} \in G^{s}\left(\Sigma_{T}\right)$. Moreover, taking into account (5.32) and (5.35), we see that $\left.(\mathbf{u}-\nabla p)\right|_{\Sigma} \in \widehat{G}^{s}\left(\Sigma_{T}\right)$. Therefore, the extension operator for $\left.(\mathbf{u}-\nabla p)\right|_{\Sigma}$ can be defined by Theorem 5.1. This and (5.40) prove the theorem.

\section{INHOMOGENEOUS BOUNDARY VALUE PROBLEM FOR THE 3D Stokes AND NAVIER-Stokes EQUations}

Let $\Omega \subset \mathbb{R}^{3}$ be a bounded domain with $C^{\infty}$-boundary $\partial \Omega$. On the cylinder $Q_{T}$, we consider the nonhomogeneous boundary value problem for the Stokes equations:

$$
\begin{gathered}
\partial_{t} \mathbf{v}-\Delta \mathbf{v}+\nabla p=\mathbf{f}, \quad \operatorname{div} \mathbf{v}=0, \quad \text { on } Q_{T}, \\
\left.\mathbf{v}\right|_{\Sigma_{T}}=\mathbf{g},
\end{gathered}
$$


and

$$
\left.\mathbf{v}\right|_{t=0}=\mathbf{v}_{0},
$$

where $\mathbf{v}$ is the velocity vector field of a fluid flow, $\nabla p$ is the pressure gradient, $\mathbf{f}$ is a given vector field (the body force per unit volume), $\mathbf{v}_{0}$ is a given initial vector, and $\mathrm{g}$ is a given Dirichlet boundary condition. Because of the divergence free condition we require that

$$
\int_{\partial \Omega} \mathbf{u}\left(t, \mathbf{x}^{\prime}\right) \cdot \mathbf{n}\left(\mathbf{x}^{\prime}\right) d \mathbf{x}^{\prime}=0 \quad \text { a.e. } t \in[0, T] .
$$

We assume that $\operatorname{div} \mathbf{v}_{0}=0$ and that the following compatibility condition holds for the boundary condition $\mathbf{g}$ and initial condition $\mathbf{v}_{0}$ :

$$
\left.\left(\mathbf{v}_{0} \cdot \mathbf{n}\right)\right|_{\partial \Omega}=\left.(\mathbf{g} \cdot \mathbf{n})\right|_{t=0} .
$$

We suppose that

$$
\begin{gathered}
\mathbf{f} \in L^{2}\left(0, T ; \mathbf{V}^{s-2}(\Omega)\right), \quad \mathbf{v}_{0} \in \mathbf{V}^{s-1}(\Omega), \\
\mathbf{g} \in G^{s}\left(\Sigma_{T}\right) \quad \text { for some } s \geq 1 .
\end{gathered}
$$

(Recall that the spaces $\mathbf{V}^{s}(\Omega)$ and $G^{s}\left(\Sigma_{T}\right)$ were introduced in Section 2.) As is well known (see [12]),

$$
\text { if } \mathbf{v}_{0} \in \mathbf{V}^{s-1}(\Omega) \text { for } s \geq 1 \text {, then }\left.\left(\mathbf{v}_{0} \cdot \mathbf{n}\right)\right|_{\partial \Omega} \in H^{s-3 / 2}(\partial \Omega) .
$$

On the other hand, the following assertion holds:

Lemma 6.1. Let $\gamma_{0} \phi=\left.\phi\right|_{t=0}$ for $\phi \in G_{n}^{s}\left(\Sigma_{T}\right)$. Then, for $s>1 / 2$ the operator

$$
\gamma_{0}: G_{n}^{s}\left(\Sigma_{T}\right) \rightarrow H^{s-3 / 2}(\partial \Omega)
$$

is well defined and continuous.

One can prove Lemma 6.1 by the techniques of Section 3 . We omit the details. By (6.7)-(6.8), the relation (6.5) is well defined for data satisfying (6.6).

For $s>3 / 2$, we impose the additional compatibility condition

$$
\left.\mathbf{v}_{0}\right|_{\partial \Omega}=\left.\mathbf{g}\right|_{t=0} .
$$

This equality is well defined because

$$
\text { if } \mathbf{v}_{0} \in \mathbf{V}^{s-1}(\Omega) \text { for } s>3 / 2 \text {, then }\left.\mathbf{v}_{0}\right|_{\partial \Omega} \in H^{s-3 / 2}(\partial \Omega)
$$

and because the following assertion is valid:

Lemma 6.2. Let $\gamma_{0} \phi=\left.\phi\right|_{t=0}$ for $\phi \in G^{s}\left(\Sigma_{T}\right)$. Then, for $s>3 / 2$, the operator

$$
\gamma_{0}: G^{s}\left(\Sigma_{T}\right) \rightarrow H^{s-3 / 2}(\partial \Omega)
$$

is well defined and continuous.

Again, this lemma can be proved by the techniques of Section 3, and we omit the details.

Theorem 6.1. Let $s \in[1,2], s \neq 3 / 2$, and assume that the data $\mathbf{f}, \mathbf{v}_{0}$, and $\mathbf{g}$ satisfy (6.4)-(6.6) and that if $s>3 / 2,(6.9)$ also holds. Then, there exists a unique solution $(\mathbf{v}, \nabla p) \in \mathcal{V}^{(s)}\left(Q_{T}\right) \times L^{2}\left(0, T ; \mathbf{H}^{s-2}(\Omega)\right)$ for the problem $(6.1)-(6.3)$. 
Proof. By virtue of Theorem 2.2, for a given Dirichlet boundary condition $\mathbf{g} \in$ $G^{s}\left(\Sigma_{T}\right)$, one can construct its extension $\mathbf{v}_{1} \in \mathcal{V}^{(s)}\left(Q_{T}\right)$ defined on $Q_{T}$ with its support in a neighborhood of $\Sigma_{T}$. We seek a solution $\mathbf{v}$ for (6.1)-(6.3) of the form

$$
\mathbf{v}=\mathbf{v}_{1}+\mathbf{w} .
$$

By substituting (6.10) into (6.1)-(6.3), we see that $\mathbf{w}$ satisfies the problem

$$
\begin{aligned}
& \partial_{t} \mathbf{w}-\Delta \mathbf{w}+\nabla p=\mathbf{f}_{1}, \quad \operatorname{div} \mathbf{w}=0, \quad \text { on } Q_{T}, \\
& \left.\mathbf{w}\right|_{\Sigma_{T}}=\mathbf{0},\left.\quad \mathbf{w}\right|_{t=0}=\mathbf{v}_{0}-\left.\mathbf{v}_{1}\right|_{t=0},
\end{aligned}
$$

where $\mathbf{f}_{1}=\mathbf{f}-\partial_{t} \mathbf{v}_{1}+\Delta \mathbf{v}_{1} \in L^{2}\left(0, T ; \mathbf{H}^{s-2}(\Omega)\right)$. As is well known (see [4], [8], and [12]), the problem (6.11) with a homogeneous boundary condition has a unique solution $(\mathbf{w}, \nabla p) \in \mathcal{V}^{(s)}\left(Q_{T}\right) \times L^{2}\left(0, T ; \mathbf{H}^{s-2}(\Omega)\right)$.

Let us consider now the inhomogeneous Dirichlet boundary value problem for the Navier-Stokes equations:

$$
\begin{gathered}
\partial_{t} \mathbf{v}-\Delta \mathbf{v}+(\mathbf{v}, \nabla) \mathbf{v}+\nabla p=\mathbf{f}, \quad \operatorname{div} \mathbf{v}=0, \quad \text { on } Q_{T}, \\
\left.\mathbf{v}\right|_{\Sigma_{T}}=\mathbf{g}, \quad \text { and }\left.\quad \mathbf{v}\right|_{t=0}=\mathbf{v}_{0} .
\end{gathered}
$$

Theorem 6.2. Let $s \in(3 / 2,2]$ and assume that the data $\mathbf{f}, \mathbf{v}_{0}$, and $\mathbf{g}$ satisfy (6.4)(6.6) and (6.9). Suppose also that $\|\mathbf{f}\|_{L^{2}\left(0, T ; H^{s-2}(\Omega)\right)}+\left\|\mathbf{v}_{0}\right\|_{V^{s-1}(\Omega)}+\|\mathbf{g}\|_{G^{s}\left(\Sigma_{T}\right)}$ is sufficiently small. Then, there exists a unique solution $(\mathbf{v}, \nabla p) \in \mathcal{V}^{(s)}\left(Q_{T}\right) \times$ $L^{2}\left(0, T ; \mathbf{H}^{s-2}(\Omega)\right)$ for the problem (6.12)-(6.13).

Proof. As in the proof of Theorem 6.1, we construct an extension $\mathbf{v}_{1} \in \mathcal{V}^{(s)}\left(Q_{T}\right)$ for the boundary data $\mathbf{g}$. Then, we look for a solution $\mathbf{v}$ in the form of (6.10). We easily see that the proof of this theorem is reduced to the proof of the existence and uniqueness of the solution $(\mathbf{w}, \nabla p) \in \mathcal{V}^{(s)}\left(Q_{T}\right) \times L^{2}\left(0, T ; \mathbf{H}^{s-2}(\Omega)\right)$ to the problem

$$
\begin{gathered}
\partial_{t} \mathbf{w}-\Delta \mathbf{w}+\left[\left(\mathbf{v}_{1}+\mathbf{w}\right) \cdot \nabla\right] \mathbf{w}+(\mathbf{w} \cdot \nabla) \mathbf{v}_{1}-\nabla p=\mathbf{f}_{2}, \\
\operatorname{div} \mathbf{w}=0, \quad \text { on } Q_{T}, \\
\left.\quad \mathbf{w}\right|_{\Sigma_{T}}=\mathbf{0},\left.\quad \mathbf{w}\right|_{t=0}=\mathbf{v}_{0}-\left.\mathbf{v}_{a}\right|_{t=0},
\end{gathered}
$$

where $\mathbf{f}_{2}=\mathbf{f}-\partial_{t} \mathbf{v}_{1}+\Delta \mathbf{v}_{1}-\left(\mathbf{v}_{1} \cdot \nabla\right) \mathbf{v}_{1} \in L^{2}\left(0, T ; \mathbf{H}^{s-2}(\Omega)\right)$. The proof of the existence of a unique solution $(\mathbf{w}, \nabla p) \in \mathcal{V}^{(s)}\left(Q_{T}\right) \times L^{2}\left(0, T ; \mathbf{H}^{s-2}(\Omega)\right)$ for (6.14)(6.15) can be realized as in [4], 8 , and $[12$.

Remark 6.1. Theorems 6.1 and 6.2 for $s>2$ are also true under suitable compatibility conditions.

\section{ACKNOWLEDGMENTS}

A. V. Fursikov was supported by the Department of Mathematics at Iowa State University. M. D. Gunzburger was supported by the National Science Foundation under grant number 9806358. L. S. Hou was supported by the Natural Science and Engineering Research Council of Canada under grant number OGP-0137436. The authors also thank the reviewers for their corrections and useful suggestions concerning previous versions of this paper. 


\section{REFERENCES}

[1] de Rham, G.; Variétés Différentiables, Hermann, Paris, 1960. MR 16:957b (1st ed.)

[2] Dubrovin, B., Fomenko, A., and Novikov, S.; Modern Geometry: Methods and Applications; Part 1. The Geometry of Surfaces, Transformation Groups, and Fields, Second Edition, Springer, New York, 1992. MR 92h:53001

[3] Foias, C. and Temam, R.; Remarques sur les équations de Navier-Stokes et les phénomènes successifs de bifurcation, Annali Scuola Norm. Sup. di Pisa Series (4) 5 1978, pp.29-63. MR 58:1749

[4] Fursikov, A.; Control problems and theorems concerning the unique solvability of mixed boundary value problems for the three dimensional Navier-Stokes and Euler equations, Math. USSR Sb. 43 1982, pp. 251-273. MR 83e:53097a

[5] Fursikov, A., Gunzburger, M., and Hou, L.; Boundary value problems and optimal boundary control for the Navier-Stokes system: the two dimensional case, SIAM J. Control Optim. 36 1998, pp. 852-894. MR 99c:76030

[6] Gelfand, I. and Shilov, G.; Distributions and operations on them, Vol.1, Fizmatgiz, Moscow, 1958; English transl., Generalized functions. Vol. 1: Properties and operations, Academic Press, New York, 1964. MR 20:4182 MR 29:3869

[7] Hormander, L.; On the theory of general partial differential operators, Acta Math. 941955 , pp. 161-248. MR 17:853d

[8] Ladyzhenskaya, O.; The Mathematical Theory of Viscous Incompressible Flow, Gordon and Breach, New York 1963. MR 27:5034b

[9] Lions, J.-L., and Magenes, E.; Problèmes Aux Limites Non Homogènes et Applications, Vol. 1, Dunod, Paris, 1968. MR 40:512

[10] Springer, G.; Introduction to Riemann Surfaces, Addison-Wesley, Boston, 1957. MR 19:1169g

[11] Schwartz, L.; Analyse Mathematique II, Hermann, Paris, 1967. MR 37:2558b

[12] Temam, R.; Navier-Stokes Equations - Theory and Numerical Analysis, Elsevier Science Publishers B.V., Amsterdam, 1984, 3rd edition (revised). MR 86m:76003

[13] Volevich, L. and Paneyakh, B.; Certain spaces of generalized functions and embedding theorems, Russian Math. Surveys 20 1965, No.1, pp. 1-73. MR 30:5160

Department of Mechanics and Mathematics, Moscow State University, Moscow 119899, Russia

E-mail address: fursikov@dial01.msu.ru

Department of Mathematics, Iowa State University, Ames, Iowa 50011-2064

E-mail address: gunzburg@iastate.edu

Department of Mathematics, Iowa State University, Ames, Iowa 50011-2064 and Department of Mathematics and Statistics, York University, Toronto, Ontario M3J 1P3, CANADA

E-mail address: hou@math.iastate.edu 